\title{
Linguistic interval 2-tuple power aggregation operators and their applications
}

\author{
Yanli Ruan, Zheng Pei, Zhisheng Gao \\ Center for Radio Administration \& Technology Development, Xihua University, \\ Chengdu, Sichuan, 610039, China \\ E-mail: pqyz@263.net \\ Received 20 July 2012 \\ Accepted 13 November 2012
}

\begin{abstract}
In this paper, we present a linguistic interval 2-tuple representation model and new linguistic interval 2tuple aggregation operators, i.e., linguistic interval 2-tuple power average (LI2TPA) operator, linguistic interval 2-tuple weighted power average (LI2TWPA) operator and linguistic interval 2-tuple power ordered weighted average (LI2TPOWA) operator. Some desired properties of the developed operators are also studied. Moreover, we use these aggregation operators to deal with multiple attribute group decision making problems under linguistic interval 2-tuple environment. In the situations where the weighting vector of the decision makers is known, we use the LI2TWPA operator to make multiple attribute group decision. In the situations where the weighting vector of the decision makers is unknown, we use the LI2TPOWA operator to deal with multiple attribute group decision making. A numerical example is provided to show the effectiveness of our method.
\end{abstract}

Keywords: Multiple attribute group decision making; Linguistic interval 2-tuple; Linguistic interval 2tuple power average (LI2TPA) operator; Linguistic interval 2-tuple weighted power average (LI2TWPA) operator; Linguistic interval 2-tuple power ordered weighted average (LI2TPOWA) operator

\section{Introduction}

Multiple attribute group decision making is a significant research topic, which consists of finding the most desirable alternatives from a given alternative set according to the information provided by some decision makers. In the real world, there exist many decision making problems in which the assessments provided by decision makers cannot be represented precisely in a quantitative form but in a qualitative one, and thus, the use of linguistic decision making approach is necessary. In the linguistic decision analysis of a multiple criteria decision making problem, the solution scheme must be formed by three steps ${ }^{4}$ : (1) the choice of the linguistic term set with its semantic; (2) the choice of the ag- gregation operator of linguistic information; (3) the choice of the best alternatives, this step is also carried out in two phases: (a) aggregation phase of linguistic information; $(b)$ exploitation phase. For the aggregation phrase of linguistic information, linguistic aggregation operators, which are used to aggregate the linguistic performance values provided by the criteria, are need to obtain a collective linguistic performance value on the alternatives. In the recent years, a variety of linguistic aggregation operators have been developed. $\mathrm{Xu}^{23}$ classified these linguistic aggregation operators into five categories: (1) the linguistic aggregation operators which are based on linear ordering, such as the linguistic max and min operators 26,27,28,29,30, linguistic median operator $28,29,30$, linguistic weighted median opera- 
tor ${ }^{28,29,30}$; (2) the linguistic aggregation operators which are based on the extension principle, such as linguistic OWA operator ${ }^{1,2,12}$, inverse-LOWA operator ${ }^{3}$, linguistic weighted OWA operator ${ }^{13}$, these operators make computations on the fuzzy numbers that support the semantics of the linguistic labels; (3) the linguistic aggregation operators which are based on symbols ${ }^{4,7}$, these operators make computations on the indexes of the linguistic labels; (4) the linguistic aggregation operators which are based on the 2-tuple linguistic representation model, such as 2-tuple arithmetic mean operator ${ }^{5,6}, 2$-tuple OWA operator $^{5,8,9}, 2$-tuple weighted geometric averaging (TWGA) operator ${ }^{22}, 2$-tuple ordered weighted geometric averaging (TOWGA) operator ${ }^{22}$; (5) the linguistic aggregation operators which compute with words directly, such as extended ordered weighted averaging (EOWA) operator ${ }^{16}$, uncertain linguistic ordered weighted operator $10,11,17$, induced uncertain linguistic OWA operator ${ }^{14,18}$. For the linguistic aggregation operators in $(1)-(3)$, the results usually do not match any of the initial linguistic terms and some approximation processes must be developed to express the results in the initial expression domain, which produces the loss of information and the lack of precision. While those operators in (4) and (5) allow that the representation of linguistic information is continuous and can express any counting of linguistic information without any loss of information in its domain.

By taking into account the relationship among aggregated values, Yager ${ }^{31}$ developed a power average (PA) operator and a power ordered weighted average (POWA) operator which allow exact arguments to support each other in the aggregation process. Afterwards, Xu and Yager ${ }^{19}$ developed some new geometric aggregation operators, including the power-geometric (PG) operator, weighted PG operator, power-ordered-geometric (POG) operator, and power-ordered-weighted-geometric (POWG) operator. They also extended the PG and POWG operators to uncertain environments, develop an uncertain PG (UPG) operator and its weighted form, and an uncertain power-ordered-weighted-geometric (UPOWG) operator to aggregate the input arguments taking the form of interval of numerical values. $\mathrm{Xu}$ and
Wang ${ }^{23}$ presented a 2-tuple linguistic power average (2TLPA) operator, a 2-tuple linguistic weighted PA operator and a 2-tuple linguistic power ordered averaging (2TLPOWA) operator. $\mathrm{Xu}{ }^{20}$ developed a series of operators for aggregating intuitionistic fuzzy numbers, involving the intuitionistic fuzzy power weighted average (IFPWA) operator, the intuitionistic fuzzy power weighted geometric (IFPWG) operator, the intuitionistic fuzzy power ordered weighted average (IFPOWA) operator and the intuitionistic fuzzy power ordered weighted geometric (IFPOWG) operator. Xu and Cai ${ }^{21}$ developed an uncertain power weighted average (UPWA) operator and an uncertain power ordered weighted average (UPOWA) operator for aggregating interval fuzzy preference relations. Xu et al. ${ }^{24} \mathrm{de}-$ veloped some new linguistic aggregation operators, such as the linguistic power average (LPA) operator, the linguistic weighted PA operator, and the LPOWA operator. They also developed some new uncertain linguistic operators under uncertain linguistic environments, such as the ULPA operator, the uncertain linguistic weighted PA operator, and the ULPOWA operator. Zhou et al. ${ }^{33}$ proposed the generalized power average (GPA) operator and its weighted form, and the generalized power ordered weighted average (GPOWA) operator. Then, they extended these operators to uncertain environments and presented an uncertain generalized power average (UGPA) operator and its weighted form, and an uncertain generalized power ordered weighted average (UGPOWA) operator to aggregate the input arguments taking the form of interval of numerical values. They also extended the GPA operator and the GPOWA operator to intuitionistic fuzzy environment, and obtained the generalized intuitionistic fuzzy power averaging (GIFPA) operator and the generalized intuitionistic fuzzy power ordered weighted averaging (GIFPOWA) operator. Zhou and Chen ${ }^{34}$ developed the generalized power average (GPA) operator and the generalized power ordered weighted average (GPOWA) operator. Then they extended the GPA operator and the GPOWA operator to linguistic environment and presented the linguistic generalized power average (LGPA) operator, the weighted linguistic generalized power average 
(WLGPA) operator and the linguistic generalized power ordered weighted average (LGPOWA) operator. Wan ${ }^{15}$ developed four kinds of power aggregation operators of trapezoidal intuitionistic fuzzy numbers (TrIFNs), involving the power average operator of TrIFNs, the weighted power average operator of TrIFNs, the power ordered weighted average operator of TrIFNs, and the power hybrid average operator of TrIFNs.

Recently, Zhang ${ }^{32}$ proposed the interval-valued 2-tuple linguistic variable which provides a tool to model qualitative information in real-world decision situations where decision makers have difficulty in giving the assessment with only a linguistic term, i.e., suppose a linguistic term set $S=$ $\left\{s_{i} \mid i=0,1,2, \ldots, g\right\}$, an interval-valued 2-tuple is composed of two linguistic terms and two numbers, denoted by $\left[\left(s_{i}, \alpha_{1}\right),\left(s_{j}, \alpha_{2}\right)\right]$, where $i \leqslant j$, $s_{i}\left(s_{j}\right)$ and $\alpha_{1}\left(\alpha_{2}\right)$ represent the linguistic label of the predefined linguistic term set $\mathrm{S}$ and symbolic translation, respectively. An interval-valued 2-tuple linguistic variable can be converted into an interval value $\left[\beta_{1}, \beta_{2}\right]\left(\beta_{1}, \beta_{2} \in[0,1], \beta_{1} \leqslant \beta_{2}\right)$ as follows: $\triangle^{-1}\left(\left[\left(s_{i}, \alpha_{1}\right),\left(s_{j}, \alpha_{2}\right)\right]\right)=\left[i / g+\alpha_{1}, j / g+\right.$ $\left.\alpha_{2}\right]=\left[\beta_{1}, \beta_{2}\right]$. On the contrary, the interval value $\left[\beta_{1}, \beta_{2}\right]\left(\beta_{1}, \beta_{2} \in[0,1], \beta_{1} \leqslant \beta_{2}\right)$ can be transformed into the interval-valued 2-tuple linguistic variable, i.e., $\triangle\left(\left[\beta_{1}, \beta_{2}\right]\right)=\left(\left[\left(s_{i}, \alpha_{1}\right),\left(s_{j}, \alpha_{2}\right)\right]\right)$, where $i=$ $\operatorname{round}\left(\beta_{1} * g\right), j=\operatorname{round}\left(\beta_{2} * g\right), \alpha_{1}=\beta_{1}-i / g$, $\alpha_{2}=\beta_{2}-j / g$, and $\alpha_{1}, \alpha_{2} \in[-0.5 / g, 0.5 / g)$. Zhang 32 think the interval-valued 2-tuple linguistic approach can aggregate the linguistic information coming from different multi-granularity linguistic term sets without unifying the multi-granularity linguistic information by the basic linguistic term set. We think it seems not so perfect. For example, suppose two linguistic term sets $S=\left\{s_{0}, s_{1}, s_{2}, s_{3}, s_{4}\right\}$ and $S^{\prime}=\left\{s_{0}^{\prime}, s_{1}^{\prime}, s_{2}^{\prime}, s_{3}^{\prime}, s_{4}^{\prime}, s_{5}^{\prime}, s_{6}^{\prime}, s_{7}^{\prime}, s_{8}^{\prime}\right\}$, an interval value $\left[\beta_{1}, \beta_{2}\right]=[0.6,0.8],\left[\beta_{1}, \beta_{2}\right]$ can be transformed into the interval-valued 2-tuple linguistic variables defined on $S$ and $S^{\prime}$, respectively. We can easily obtain $\triangle([0.6,0.8])=\left(\left[\left(s_{2}, 0.1\right),\left(s_{3}, 0.05\right)\right]\right)$ and $\triangle^{\prime}([0.6,0.8])=\left(\left[\left(s_{5}^{\prime},-0.025\right),\left(s_{6}^{\prime}, 0.05\right)\right]\right)$. In practice, it is a problem to decide whether $\left[\left(s_{2}, 0.1\right),\left(s_{3}, 0.05\right)\right]$ and $\left[\left(s_{5}^{\prime},-0.025\right),\left(s_{6}^{\prime}, 0.05\right)\right]$ are equivalent. In this paper, for a fixed linguis- tic term set, we develop a linguistic interval 2-tuple representation model for uncertainty in linguistic decision making process. We extend then the PA operator and the POWA operator to aggregate linguistic interval 2-tuples, which are called linguistic interval 2-tuple power average (LI2TPA) operator, linguistic interval 2-tuple weighted power average (LI2TWPA) operator and linguistic interval 2-tuple power ordered weighted average (LI2TPOWA) operator, whose weighting vectors depend on the aggregated arguments and allow aggregated arguments to support each other.

The rest of this paper is organized as follows: In Section 2, we briefly introduce some basic knowledge such as the 2-tuple linguistic representation model, the PA operator, the OWA operator and the POWA operator. In Section 3, we present a linguistic interval 2-tuple representation model and develop a linguistic interval 2-tuple power average (LI2TPA) operator, a linguistic interval 2-tuple power weighted average (LI2TWPA) operator and a linguistic interval 2-tuple power ordered weighted average (LI2TPOWA) operator. Some desired properties of these operators are also studied. In Section 4, we develop two new approaches based on the LI2TWPA operator and LI2TPOWA operator respectively to deal with linguistic multiple attribute group decision making problems. In Section 5, a numerical example is provided to illustrate the linguistic multiple attribute group decision making process, which shows the effectiveness of the new developed approach. Finally, conclusions are provided in Section 6.

\section{Preliminaries}

In this section, we briefly review the 2-tuple linguistic representation model, the $P A$ operator, $O W A$ operator and $P O W A$ operator.

\subsection{The 2-tuple linguistic representation model}

One of the most often used models dealing with linguistic information is the 2-tuple linguistic representation model, which was developed by Herrera and Martínez ${ }^{5}$ on the basis of the concept of symbolic 
translation. In the following, we briefly review some concepts about 2-tuple.

Let $S=\left\{s_{0}, s_{1}, s_{2}, \ldots, s_{g}\right\}$ be a linguistic term set. For example, a set of seven terms $\mathrm{S}$ could be: $S=$ $\left\{s_{0}=\right.$ very poor,$s_{1}=$ poor,$s_{2}=$ slightly poor,$s_{3}=$ fair, $s_{4}=$ slightly good,$s_{5}=$ good,$s_{6}=$ very $\left.\operatorname{good}\right\}$. Usually, it is required that the linguistic term set satisfies the following additional characteristics: (1) There is a negation operator: $\operatorname{Neg}\left(s_{i}\right)=s_{j}$ such that $j=g-i(g+1$ is the cardinality of $S)$; (2) $s_{i} \leqslant s_{j} \Leftrightarrow i \leqslant j$. Therefore, there exists a minimization and a maximization operator in the linguistic term set $S$.

Definition 1. ${ }^{5}$ Let $\beta$ be the result of an aggregation of the indexes of a set of labels assessed in a linguistic term set $S$, i.e., the result of a symbolic aggregation operation. $\beta \in[0, g]$, being $g+1$ the cardinality of $S$. Let $i=\operatorname{round}(\beta)$ and $\alpha=\beta-i$ be two values such that $i \in[0, g]$ and $\alpha \in[-0.5,0.5)$, then $\alpha$ is called a symbolic translation.

From the concept of symbolic translation, Herrera and Martínez developed a linguistic representation model which represents the linguistic information by means of 2-tuple $\left(s_{i}, \alpha_{i}\right), s_{i} \in S$ and $\alpha_{i} \in$ $[-0.5,0.5), s_{i}$ represents the linguistic label center of the information, $\alpha_{i}$ is a numerical value expressing the value of the translation from the original result $\beta$ to the closest index label, $i$, in the linguistic term set $\left(s_{i} \in S\right)$, i.e., the symbolic translation.

This model defines a set of transformation functions between linguistic terms and 2-tuples and between numerical values and 2-tuples.

Definition 2. ${ }^{5}$ Let $S=\left\{s_{0}, s_{1}, s_{2}, \ldots, s_{g}\right\}$ be a linguistic term set and $\beta \in[0, g]$ be a value representing the result of a symbolic aggregation operation, then the 2-tuple that expresses the equivalent information to $\beta$ is obtained with the following function:

$$
\begin{aligned}
\triangle:[0, g] & \rightarrow S \times[-0.5,0.5) \\
\beta & \longmapsto \triangle(\beta)=\left(s_{i}, \alpha\right)
\end{aligned}
$$

where $s_{i} \in S, i=\operatorname{round}(\beta)$ (round is the usual round operation), $\alpha=\beta-i \in[-0.5,0.5)$.

On the contrary, Let $S=\left\{s_{0}, s_{1}, s_{2}, \ldots, s_{g}\right\}$ be a linguistic term set and $\left(s_{i}, \alpha\right)$ be a 2 -tuple. There is always a $\triangle^{-1}$ function:

$$
\begin{array}{r}
\triangle^{-1}: S \times[-0.5,0.5) \rightarrow[0, g] \\
\triangle^{-1}\left(s_{i}, \alpha\right)=i+\alpha=\beta
\end{array}
$$

Remark 1. From Definitions 1 and 2, it is obvious that the conversion of a linguistic term into a linguistic 2-tuple consists of adding a value 0 as symbolic translation:

$$
s_{i} \in S \Rightarrow\left(s_{i}, 0\right)
$$

Comparison of 2-Tuples: let $\left(s_{k}, \alpha_{k}\right)$ and $\left(s_{l}, \alpha_{l}\right)$ be two 2-tuples, with each one representing a counting of information as follows:

1. If $k<l$, then $\left(s_{k}, \alpha_{k}\right)<\left(s_{l}, \alpha_{l}\right)$;

2. If $k=l$, then:

(a) if $\alpha_{k}<\alpha_{l}$, then $\left(s_{k}, \alpha_{k}\right)<\left(s_{l}, \alpha_{l}\right)$;

(b) if $\alpha_{k}=\alpha_{l}$, then $\left(s_{k}, \alpha_{k}\right)=\left(s_{l}, \alpha_{l}\right)$.

\subsection{The PA operator, the OWA operator and the POWA operator}

Most of the existing aggregation operators do not take into account the information about the relationship between the values being aggregated. Thus, Yager $^{31}$ developed a power average $(P A)$ operator which allows that the aggregated values support and reinforce each other in the decision making process. The PA operator was defined as follows:

$$
P A\left(a_{1}, a_{2}, \ldots, a_{n}\right)=\frac{\sum_{i=1}^{n}\left(1+T\left(a_{i}\right)\right) a_{i}}{\sum_{i=1}^{n}\left(1+T\left(a_{i}\right)\right)}
$$

where

$$
T\left(a_{i}\right)=\sum_{\substack{j=1 \\ j \neq i}}^{n} \operatorname{Sup}\left(a_{i}, a_{j}\right)
$$

and $\operatorname{Sup}(a, b)$ is the support for $a$ from $b$, which satisfies the following three properties:

1) $\operatorname{Sup}(a, b) \in[0,1]$.

2) $\operatorname{Sup}(a, b)=\operatorname{Sup}(b, a)$.

3) $\operatorname{Sup}(a, b) \geqslant \operatorname{Sup}(x, y)$, if $|a-b|<|x-y|$.

Obviously, the support (i.e., Sup) measure is essentially a similarity index. The more the similarity, 
the closer the two values are, and the more they support each other.

Yager $^{25}$ developed an OWA operator that provides an aggregation lying between the max and min operators, the OWA operator was defined as follows:

$$
O W A\left(a_{1}, a_{2}, \ldots, a_{n}\right)=\sum_{i=1}^{n} w_{i} b_{i}
$$

where $b_{i}$ is the $i$ th largest argument of $b_{j}(j=$ $1,2, \ldots, n)$.

Based on the OWA and PA operators, Yager ${ }^{31}$ defined a POWA operator as follows:

$$
\operatorname{POWA}\left(a_{1}, a_{2}, \ldots, a_{n}\right)=\sum_{i=1}^{n} u_{i} a_{i n d e x(i)}
$$

where $u_{i}=g\left(\frac{R_{i}}{T V}\right)-g\left(\frac{R_{i-1}}{T V}\right), \quad R_{i}=\sum_{j=1}^{i} V_{V_{\text {index }(j)}}$, $T V=\sum_{i=1}^{n} V_{\text {index }(i)}, \quad V_{\text {index }(i)}=1+T\left(a_{\text {index }(i)}\right)$ and $T\left(a_{\text {index }(i)}\right)=\sum_{j=1, j \neq i}^{n} \operatorname{Sup}\left(a_{\text {index }(i)}, a_{\text {index }(j)}\right)$, $T\left(a_{\text {index }(i)}\right)$ denotes the support of the $i$ th largest argument by all the other arguments, $\operatorname{Sup}\left(a_{\text {index }(i)}, a_{\text {index }(j)}\right)$ indicates the support of $j$ th largest argument for the $i$ th largest argument, $a_{\text {index }(i)}$ is the $i$ th largest argument of the arguments $a_{j}(j=1,2, \ldots, n)$ and $g:[0,1] \rightarrow[0,1]$ is a basic unit interval monotonic (BUM) function, having the properties: 1) $g(0)=0$; 2) $g(1)=1$; 3) $g(x) \geqslant g(y)$, if $x>y$.

Especially, if $g(x)=x$, then the POWA operator reduces to the PA operator.

\section{The linguistic interval 2-tuple representation model}

Due to the complexity of the objects and the vagueness of inherent subjective nature of human thinking, there are many decision making problems in which the linguistic information provided by decision makers may take the form of interval, denoted by $\left[s_{i}, s_{j}\right]$, with $s_{i}, s_{j} \in S$ and $s_{i}<s_{j}, S=$ $\left\{s_{0}, s_{1}, \ldots, s_{g}\right\}$ is the predefined linguistic term set. Recently, Zhang ${ }^{32}$ proposed the interval-valued 2tuple linguistic variable and the procedure to compare two interval-valued 2-tuples. Motivated by Zhang and considering the advantage of the 2-tuple linguistic representation model, we propose the linguistic interval 2-tuple representation model to deal with the above decision making problems, which can be described as follows.

Let $S=\left\{s_{0}, s_{1}, s_{2}, \ldots, s_{g}\right\}$ be a linguistic term set. A linguistic interval 2-tuple is composed of two linguistic terms and two numbers, denoted by $\left[\left(s_{i}, \alpha_{1}\right),\left(s_{j}, \alpha_{2}\right)\right]$, where $i \leqslant j, s_{i}\left(s_{j}\right)$ and $\alpha_{1}\left(\alpha_{2}\right)$ represent the linguistic label of the predefined linguistic term set $\mathrm{S}$ and symbolic translation, respectively. A linguistic interval 2-tuple can be converted into an interval number $\left[\beta_{1}, \beta_{2}\right]\left(\beta_{1}, \beta_{2} \in[0, g], \beta_{1} \leqslant\right.$ $\beta_{2}$ ) as follows:

$$
\triangle^{-1}\left(\left[\left(s_{i}, \alpha_{1}\right),\left(s_{j}, \alpha_{2}\right)\right]\right)=\left[i+\alpha_{1}, j+\alpha_{2}\right]=\left[\beta_{1}, \beta_{2}\right] .
$$

On the contrary, an interval number $\left[\beta_{1}, \beta_{2}\right]\left(\beta_{1}, \beta_{2} \in[0, g], \beta_{1} \leqslant \beta_{2}\right)$ can be transformed into a linguistic interval 2 -tuple by the following function:

$$
\begin{gathered}
\triangle\left(\left[\beta_{1}, \beta_{2}\right]\right)=\left[\left(s_{i}, \alpha_{1}\right),\left(s_{j}, \alpha_{2}\right)\right], \\
\text { with }\left\{\begin{array}{ll}
s_{i}, & i=\operatorname{round}\left(\beta_{1}\right) \\
s_{j}, & j=\operatorname{round}\left(\beta_{2}\right) \\
\alpha_{1}=\beta_{1}-i, & \alpha_{1} \in[-0.5,0.5) \\
\alpha_{2}=\beta_{2}-j, & \alpha_{2} \in[-0.5,0.5)
\end{array} .\right.
\end{gathered}
$$

The negation operator over the linguistic interval 2-tuples is defined as follows:

$$
\begin{aligned}
& N e g\left(\left[\left(s_{i}, \alpha_{1}\right),\left(s_{j}, \alpha_{2}\right)\right]\right) \\
& =\triangle\left(\left[1-\triangle^{-1}\left(s_{j}, \alpha_{2}\right), 1-\triangle^{-1}\left(s_{i}, \alpha_{1}\right)\right]\right) .
\end{aligned}
$$

Specially, if $s_{i}=s_{j}$ and $\alpha_{1}=\alpha_{2}$, then the linguistic interval 2-tuple representation model reduces to the 2-tuple linguistic representation model.

To compare linguistic interval 2-tuples, we propose the score and accuracy functions as follows.

Definition 3. For a linguistic interval 2-tuple $A=$ $\left[\left(s_{i}, \alpha_{1}\right),\left(s_{j}, \alpha_{2}\right)\right]$, its score function is expressed as follows:

$$
S(A)=(i+j) / 2+\left(\alpha_{1}+\alpha_{2}\right) / 2 .
$$


It is obvious that $0 \leqslant S(A) \leqslant g$. The score function is regarded as a basis to compare two linguistic interval 2-tuples. For two linguistic interval 2tuples, the one with a larger score function corresponds to the larger linguistic interval 2-tuple. However, it is really possible that two different linguistic interval 2-tuples may have an identical score value. In such a case, the accuracy function should be taken into account.

Definition 4. For a linguistic interval 2-tuple $A=$ $\left[\left(s_{i}, \alpha_{1}\right),\left(s_{j}, \alpha_{2}\right)\right]$, its accuracy function is expressed as follows:

$$
H(A)=(j-i)+\left(\alpha_{2}-\alpha_{1}\right)
$$

It is easy to prove that $0 \leqslant H(A) \leqslant g$. For two linguistic interval 2-tuples with the same score function, the smaller the accuracy function, the larger the corresponding linguistic interval 2-tuple.

Based on the score function and accuracy function, the procedure to compare two linguistic interval 2-tuples is listed as follows.

Let $A=\left[\left(s_{i}, \alpha_{1}\right),\left(s_{j}, \alpha_{2}\right)\right]$ and $B=$ $\left[\left(s_{i}^{*}, \alpha_{1}^{*}\right),\left(s_{j}^{*}, \alpha_{2}^{*}\right)\right]$ be two linguistic interval 2-tuples:

1. If $S(A)<S(B)$, then $A<B$;

2. If $S(A)=S(B)$, then:

(a) if $H(A)<H(B)$, then $A>B$;

(b) if $H(A)=H(B)$, then $A=B$.

Intuitively, the score function and the accuracy function of a linguistic interval 2-tuple denote the center and the width of the linguistic interval 2tuple, respectively. For two linguistic interval 2tuples, the larger the center, the larger the corresponding linguistic interval 2-tuple. If two linguistic interval 2-tuples have the same center, the larger the width, the smaller the corresponding linguistic interval 2-tuple.

\section{Linguistic interval 2-tuple power aggregation operators}

Based on the PA operator ${ }^{31}$ and the above linguistic interval 2-tuple representation model, we define a LI2TPA operator as follows.
Definition 5. Let $X=\left\{\left[\left(s_{1}, \alpha_{1}\right),\left(s_{1^{\prime}}, \alpha_{1^{\prime}}\right)\right], \ldots,\left[\left(s_{n}\right.\right.\right.$, $\left.\left.\left.\alpha_{n}\right),\left(s_{n^{\prime}}, \alpha_{n^{\prime}}\right)\right]\right\}$ be a set of linguistic interval 2tuples. The linguistic interval 2-tuple power average (LI2TPA) operator is defined as

$$
\begin{aligned}
& \operatorname{LI2TPA}\left(\left[\left(s_{1}, \alpha_{1}\right),\left(s_{1^{\prime}}, \alpha_{1^{\prime}}\right)\right], \ldots,\left[\left(s_{n}, \alpha_{n}\right),\left(s_{n^{\prime}}, \alpha_{n^{\prime}}\right)\right]\right) \\
& =\triangle\left(\frac{\sum_{j=1}^{n}(1+M) \triangle^{-1}\left(\left[\left(s_{j}, \alpha_{j}\right),\left(s_{j^{\prime}}, \alpha_{j^{\prime}}\right)\right]\right)}{\sum_{j=1}^{n}(1+M)}\right)
\end{aligned}
$$

where $M=T\left(\left[\left(s_{j}, \alpha_{j}\right),\left(s_{j^{\prime}}, \alpha_{j^{\prime}}\right)\right]\right)=\sum_{i=1, i \neq j}^{n} \operatorname{Sup}([$ $\left.\left.\left(s_{j}, \alpha_{j}\right),\left(s_{j^{\prime}}, \alpha_{j^{\prime}}\right)\right],\left[\left(s_{i}, \alpha_{i}\right),\left(s_{i^{\prime}}, \alpha_{i^{\prime}}\right)\right]\right)$ and $\operatorname{Sup}\left(\left[\left(s_{j}\right.\right.\right.$, $\left.\left.\left.\alpha_{j}\right),\left(s_{j^{\prime}}, \alpha_{j^{\prime}}\right)\right], \quad\left[\left(s_{i}, \alpha_{i}\right),\left(s_{i^{\prime}}, \alpha_{i^{\prime}}\right)\right]\right)$ is the support for the linguistic interval 2-tuple $\left[\left(s_{j}, \alpha_{j}\right),\left(s_{j^{\prime}}, \alpha_{j^{\prime}}\right)\right]$ from $\left[\left(s_{i}, \alpha_{i}\right),\left(s_{i^{\prime}}, \alpha_{i^{\prime}}\right)\right]$, which satisfies the following three properties: (1) $\operatorname{Sup}\left(\left[\left(s_{j}, \alpha_{j}\right),\left(s_{j^{\prime}}, \alpha_{j^{\prime}}\right)\right],\left[\left(s_{i}, \alpha_{i}\right),\left(s_{i^{\prime}}, \alpha_{i^{\prime}}\right)\right]\right) \in[0,1] ;$ (2) $\operatorname{Sup}\left(\left[\left(s_{j}, \alpha_{j}\right),\left(s_{j^{\prime}}, \alpha_{j^{\prime}}\right)\right],\left[\left(s_{i}, \alpha_{i}\right),\left(s_{i^{\prime}}, \alpha_{i^{\prime}}\right)\right]\right)=\operatorname{Sup}$ $\left(\left[\left(s_{i}, \alpha_{i}\right),\left(s_{i^{\prime}}, \alpha_{i^{\prime}}\right)\right],\left[\left(s_{j}, \alpha_{j}\right),\left(s_{j^{\prime}}, \alpha_{j^{\prime}}\right)\right]\right)$;

(3) $\operatorname{Sup}\left(\left[\left(s_{i}, \alpha_{i}\right),\left(s_{i^{\prime}}, \alpha_{i^{\prime}}\right)\right],\left[\left(s_{j}, \alpha_{j}\right),\left(s_{j^{\prime}}, \alpha_{j^{\prime}}\right)\right]\right) \geqslant$ $\operatorname{Sup}\left(\left[\left(s_{k}, \alpha_{k}\right),\left(s_{k^{\prime}}, \alpha_{k^{\prime}}\right)\right],\left[\left(s_{l}, \alpha_{l}\right),\left(s_{l^{\prime}}, \alpha_{l^{\prime}}\right)\right]\right)$, if, $d\left(\left[\left(s_{i}\right.\right.\right.$, $\left.\left.\left.\alpha_{i}\right),\left(s_{i^{\prime}}, \alpha_{i^{\prime}}\right)\right],\left[\left(s_{j}, \alpha_{j}\right),\left(s_{j^{\prime}}, \alpha_{j^{\prime}}\right)\right]\right)<d\left(\left[\left(s_{k}, \alpha_{k}\right),\left(s_{k^{\prime}}\right.\right.\right.$, $\left.\left.\left.\alpha_{k^{\prime}}\right)\right],\left[\left(s_{l}, \alpha_{l}\right),\left(s_{l^{\prime}}, \alpha_{l^{\prime}}\right)\right]\right)$, where $d$ is a distance measure for linguistic interval 2-tuples.

Obviously, the support measure is essentially a similarity index, i.e., the closer the two linguistic interval 2-tuples are, then the more the support measure is, the more their similarity is.

In the following, we discuss some desired properties of the LI2TPA operator.

Theorem 1. Let $\operatorname{Sup}\left(\left[\left(s_{i}, \alpha_{i}\right),\left(s_{i^{\prime}}, \alpha_{i^{\prime}}\right)\right],\left[\left(s_{j}, \alpha_{j}\right),\left(s_{j^{\prime}}\right.\right.\right.$, $\left.\left.\left.\alpha_{j^{\prime}}\right)\right]\right)=k$, for all $i \neq j$, then

$$
\begin{aligned}
& \operatorname{LI2TPA}\left(\left[\left(s_{1}, \alpha_{1}\right),\left(s_{1^{\prime}}, \alpha_{1^{\prime}}\right)\right], \ldots,\left[\left(s_{n}, \alpha_{n}\right),\left(s_{n^{\prime}}, \alpha_{n^{\prime}}\right)\right]\right) \\
& =\triangle\left(\frac{1}{n} \sum_{j=1}^{n} \triangle^{-1}\left(\left[\left(s_{j}, \alpha_{j}\right),\left(s_{j^{\prime}}, \alpha_{j^{\prime}}\right)\right]\right)\right)
\end{aligned}
$$

which indicates that when all the supports are the same, the LI2TPA operator is simply a linguistic interval 2-tuple average operator.

Proof. If $\operatorname{Sup}\left(\left[\left(s_{i}, \alpha_{i}\right),\left(s_{i^{\prime}}, \alpha_{i^{\prime}}\right)\right],\left[\left(s_{j}, \alpha_{j}\right),\left(s_{j^{\prime}}, \alpha_{j^{\prime}}\right)\right]\right)$ $=k$, for all $i \neq j$, then

$$
T\left(\left[\left(s_{j}, \alpha_{j}\right),\left(s_{j^{\prime}}, \alpha_{j^{\prime}}\right)\right]\right)=(n-1) k
$$


Thus

$$
\begin{aligned}
& \operatorname{LI2TPA}\left(\left[\left(s_{1}, \alpha_{1}\right),\left(s_{1^{\prime}}, \alpha_{1^{\prime}}\right)\right], \ldots,\left[\left(s_{n}, \alpha_{n}\right),\left(s_{n^{\prime}}, \alpha_{n^{\prime}}\right)\right]\right) \\
& =\triangle\left(\frac{\sum_{j=1}^{n}(1+(n-1) k) \triangle^{-1}\left(\left[\left(s_{j}, \alpha_{j}\right),\left(s_{j^{\prime}}, \alpha_{j^{\prime}}\right)\right]\right)}{\sum_{j=1}^{n}(1+(n-1) k)}\right) \\
& =\triangle\left(\frac{1}{n} \sum_{j=1}^{n} \triangle^{-1}\left(\left[\left(s_{j}, \alpha_{j}\right),\left(s_{j^{\prime}}, \alpha_{j^{\prime}}\right)\right]\right)\right)
\end{aligned}
$$

which is simply a linguistic interval 2-tuple average operator.

Especially, if $\operatorname{Sup}\left(\left[\left(s_{i}, \alpha_{i}\right),\left(s_{i^{\prime}}, \alpha_{i^{\prime}}\right)\right],\left[\left(s_{j}, \alpha_{j}\right),\left(s_{j^{\prime}}\right.\right.\right.$, $\left.\left.\left.\alpha_{j^{\prime}}\right)\right]\right)=0$, for all $i \neq j$, i.e., all the supports are zero, then there is no support in the LI2TPA, and in this case, we have $T\left(\left[\left(s_{j}, \alpha_{j}\right),\left(s_{j^{\prime}}, \alpha_{j^{\prime}}\right)\right]\right)=0, j=$ $1,2, \ldots, n$, and $\sum_{j=1}^{n}\left(1+T\left(\left[\left(s_{j}, \alpha_{j}\right),\left(s_{j^{\prime}}, \alpha_{j^{\prime}}\right)\right]\right)\right)=n$. By Eq.(1), it is clear that the LI2TPA operator reduces to the LI2TA operator.

Theorem 2. (Commutativity) Let $\left\{\left[\left(s_{1}, \alpha_{1}\right),\left(s_{1^{\prime}}, \alpha_{1^{\prime}}\right)\right]\right.$, $\left.\ldots,\left[\left(s_{n}, \alpha_{n}\right),\left(s_{n^{\prime}}, \alpha_{n^{\prime}}\right)\right]\right\}$ and $\left\{\left[\left(s_{1}^{*}, \alpha_{1}^{*}\right),\left(s_{1^{\prime}}^{*}, \alpha_{1^{\prime}}^{*}\right)\right], \ldots\right.$, $\left.\left[\left(s_{n}^{*}, \alpha_{n}^{*}\right),\left(s_{n^{\prime}}^{*}, \alpha_{n^{\prime}}^{*}\right)\right]\right\}$ be two collections of linguistic interval 2-tuples, then

$$
\begin{aligned}
& \operatorname{LI} 2 \operatorname{TPA}\left(\left[\left(s_{1}, \alpha_{1}\right),\left(s_{1^{\prime}}, \alpha_{1^{\prime}}\right)\right], \ldots,\left[\left(s_{n}, \alpha_{n}\right),\left(s_{n^{\prime}}, \alpha_{n^{\prime}}\right)\right]\right) \\
& =\operatorname{LI} 2 \operatorname{TPA}\left(\left[\left(s_{1}^{*}, \alpha_{1}^{*}\right),\left(s_{1^{\prime}}^{*}, \alpha_{1^{\prime}}^{*}\right)\right], \ldots,\left[\left(s_{n}^{*}, \alpha_{n}^{*}\right),\left(s_{n^{\prime}}^{*}, \alpha_{n^{\prime}}^{*}\right)\right]\right)
\end{aligned}
$$

where $\left\{\left[\left(s_{1}^{*}, \alpha_{1}^{*}\right),\left(s_{1^{\prime}}^{*}, \alpha_{1^{\prime}}^{*}\right)\right], \ldots,\left[\left(s_{n}^{*}, \alpha_{n}^{*}\right),\left(s_{n^{\prime}}^{*}, \alpha_{n^{\prime}}^{*}\right)\right]\right\}$ is any permutation of $\left\{\left[\left(s_{1}, \alpha_{1}\right),\left(s_{1^{\prime}}, \alpha_{1^{\prime}}\right)\right], \ldots,\left[\left(s_{n}\right.\right.\right.$, $\left.\left.\left.\alpha_{n}\right),\left(s_{n^{\prime}}, \alpha_{n^{\prime}}\right)\right]\right\}$.

Proof. Let

$$
\begin{aligned}
& \operatorname{LI2TPA}\left(\left[\left(s_{1}, \alpha_{1}\right),\left(s_{1^{\prime}}, \alpha_{1^{\prime}}\right)\right], \ldots,\left[\left(s_{n}, \alpha_{n}\right),\left(s_{n^{\prime}}, \alpha_{n^{\prime}}\right)\right]\right) \\
& =\triangle\left(\frac{\sum_{j=1}^{n}(1+M) \triangle^{-1}\left(\left[\left(s_{j}, \alpha_{j}\right),\left(s_{j^{\prime}}, \alpha_{j^{\prime}}\right)\right]\right)}{\sum_{j=1}^{n}(1+M)}\right)
\end{aligned}
$$

$\operatorname{LI} 2 T P A\left(\left[\left(s_{1}^{*}, \alpha_{1}^{*}\right),\left(s_{1^{\prime}}^{*}, \alpha_{1^{\prime}}^{*}\right)\right], \ldots,\left[\left(s_{n}^{*}, \alpha_{n}^{*}\right),\left(s_{n^{\prime}}^{*}, \alpha_{n^{\prime}}^{*}\right)\right]\right)$

$$
=\triangle\left(\frac{\sum_{j=1}^{n}\left(1+M^{*}\right) \triangle^{-1}\left(\left[\left(s_{j}^{*}, \alpha_{j}^{*}\right),\left(s_{j^{\prime}}^{*}, \alpha_{j^{\prime}}^{*}\right)\right]\right)}{\sum_{j=1}^{n}\left(1+M^{*}\right)}\right)
$$

where $\quad M=T\left(\left[\left(s_{j}^{*}, \alpha_{j}^{*}\right),\left(s_{j^{\prime}}^{*}, \alpha_{j^{\prime}}^{*}\right)\right]\right), \quad M^{*}=$ $T\left(\left[\left(s_{j}^{*}, \alpha_{j}^{*}\right),\left(s_{j^{\prime}}^{*}, \alpha_{j^{\prime}}^{*}\right)\right]\right)$.

Since $\left\{\left[\left(s_{1}^{*}, \alpha_{1}^{*}\right),\left(s_{1^{\prime}}^{*}, \alpha_{1^{\prime}}^{*}\right)\right], \ldots,\left[\left(s_{n}^{*}, \alpha_{n}^{*}\right),\left(s_{n^{\prime}}^{*}, \alpha_{n^{\prime}}^{*}\right)\right]\right\}$ is any permutation of $\left\{\left[\left(s_{1}, \alpha_{1}\right),\left(s_{1^{\prime}}, \alpha_{1^{\prime}}\right)\right], \ldots,\left[\left(s_{n}, \alpha_{n}\right)\right.\right.$, $\left.\left.\left(s_{n^{\prime}}, \alpha_{n^{\prime}}\right)\right]\right\}$, then we have $M=M^{*}, \sum_{j=1}^{n}(1+M)=$ $\sum_{j=1}^{n}\left(1+M^{*}\right), \sum_{j=1}^{n}(1+M) \triangle^{-1}\left(\left[\left(s_{j}, \alpha_{j}\right),\left(s_{j^{\prime}}, \alpha_{j^{\prime}}\right)\right]\right)$ $=\sum_{j=1}^{n}\left(1+M^{*}\right) \triangle^{-1}\left(\left[\left(s_{j}^{*}, \alpha_{j}^{*}\right),\left(s_{j^{\prime}}^{*}, \alpha_{j^{\prime}}^{*}\right)\right]\right)$, thus

$\operatorname{LI2TPA}\left(\left[\left(s_{1}, \alpha_{1}\right),\left(s_{1^{\prime}}, \alpha_{1^{\prime}}\right)\right], \ldots,\left[\left(s_{n}, \alpha_{n}\right),\left(s_{n^{\prime}}, \alpha_{n^{\prime}}\right)\right]\right)$

$=\operatorname{LI} 2 \operatorname{TPA}\left(\left[\left(s_{1}^{*}, \alpha_{1}^{*}\right),\left(s_{1^{\prime}}^{*}, \alpha_{1^{\prime}}^{*}\right)\right], \ldots,\left[\left(s_{n}^{*}, \alpha_{n}^{*}\right),\left(s_{n^{\prime}}^{*}, \alpha_{n^{\prime}}^{*}\right)\right]\right)$

Theorem 3. (Idempotency) Let $\left\{\left[\left(s_{1}, \alpha_{1}\right),\left(s_{1^{\prime}}, \alpha_{1^{\prime}}\right)\right]\right.$, $\left.\ldots,\left[\left(s_{n}, \alpha_{n}\right),\left(s_{n^{\prime}}, \alpha_{n^{\prime}}\right)\right]\right\}$ be a collection of linguistic interval 2-tuples, if all $\left[\left(s_{j}, \alpha_{j}\right),\left(s_{j^{\prime}}, \alpha_{j^{\prime}}\right)\right](j=$ $1,2, \ldots, n)$ are equal, i.e., $\left[\left(s_{j}, \alpha_{j}\right),\left(s_{j^{\prime}}, \alpha_{j^{\prime}}\right)\right]=$ $\left[(s, \alpha),\left(s^{\prime}, \alpha^{\prime}\right)\right]$, for all $j$, then

$$
\begin{aligned}
& \operatorname{LI2TPA}\left(\left[\left(s_{1}, \alpha_{1}\right),\left(s_{1^{\prime}}, \alpha_{1^{\prime}}\right)\right], \ldots,\left[\left(s_{n}, \alpha_{n}\right),\left(s_{n^{\prime}}, \alpha_{n^{\prime}}\right)\right]\right) \\
& =\left[(s, \alpha),\left(s^{\prime}, \alpha^{\prime}\right)\right]
\end{aligned}
$$

Proof. If $\left[\left(s_{j}, \alpha_{j}\right),\left(s_{j^{\prime}}, \alpha_{j^{\prime}}\right)\right]=\left[(s, \alpha),\left(s^{\prime}, \alpha^{\prime}\right)\right]$, for all $j$, then

$$
\begin{aligned}
& \operatorname{LI} 2 T P A\left(\left[\left(s_{1}, \alpha_{1}\right),\left(s_{1^{\prime}}, \alpha_{1^{\prime}}\right)\right], \ldots,\left[\left(s_{n}, \alpha_{n}\right),\left(s_{n^{\prime}}, \alpha_{n^{\prime}}\right)\right]\right) \\
& =\triangle\left(\frac{\sum_{j=1}^{n}(1+M) \triangle^{-1}\left(\left[\left(s_{j}, \alpha_{j}\right),\left(s_{j^{\prime}}, \alpha_{j^{\prime}}\right)\right]\right)}{\sum_{j=1}^{n}(1+M)}\right) \\
& =\triangle\left(\frac{\sum_{j=1}^{n}(1+M)}{\sum_{j=1}^{n}\left(1+M^{*}\right)} \triangle^{-1}\left(\left[(s, \alpha),\left(s^{\prime}, \alpha^{\prime}\right)\right]\right)\right) \\
& =\left[(s, \alpha),\left(s^{\prime}, \alpha^{\prime}\right)\right]
\end{aligned}
$$

Theorem 4. (Boundedness) Let $\left\{\left[\left(s_{1}, \alpha_{1}\right),\left(s_{1^{\prime}}, \alpha_{1^{\prime}}\right)\right]\right.$, $\left.\ldots,\left[\left(s_{n}, \alpha_{n}\right),\left(s_{n^{\prime}}, \alpha_{n^{\prime}}\right)\right]\right\}$ be a collection of linguistic interval 2-tuples, then

$$
\begin{aligned}
& \min _{j}\left(\left[\left(s_{j}, \alpha_{j}\right),\left(s_{j^{\prime}}, \alpha_{j^{\prime}}\right)\right]\right) \\
& \quad \leqslant \operatorname{LI} 2 \operatorname{TPA}\left(\left[\left(s_{1}, \alpha_{1}\right),\left(s_{1^{\prime}}, \alpha_{1^{\prime}}\right)\right], \ldots,\left[\left(s_{n}, \alpha_{n}\right),\left(s_{n^{\prime}}, \alpha_{n^{\prime}}\right)\right]\right) \\
& \quad \leqslant \max _{j}\left(\left[\left(s_{j}, \alpha_{j}\right),\left(s_{j^{\prime}}, \alpha_{j^{\prime}}\right)\right]\right)
\end{aligned}
$$

Proof. $\quad$ Since $\min _{j}\left(\left[\left(s_{j}, \alpha_{j}\right),\left(s_{j^{\prime}}, \alpha_{j^{\prime}}\right)\right]\right) \leqslant$ 
$\left[\left(s_{j}, \alpha_{j}\right),\left(s_{j^{\prime}}, \alpha_{j^{\prime}}\right)\right] \leqslant \max _{j}\left(\left[\left(s_{j}, \alpha_{j}\right),\left(s_{j^{\prime}}, \alpha_{j^{\prime}}\right)\right]\right)$, then

$$
\begin{aligned}
& \triangle\left(\frac{\sum_{j=1}^{n}(1+M) \triangle^{-1}\left(\min _{j}\left(\left[\left(s_{j}, \alpha_{j}\right),\left(s_{j^{\prime}}, \alpha_{j^{\prime}}\right)\right]\right)\right)}{\sum_{j=1}^{n}(1+M)}\right) \\
& \leqslant \triangle\left(\frac{\sum_{j=1}^{n}(1+M) \triangle^{-1}\left(\left[\left(s_{j}, \alpha_{j}\right),\left(s_{j^{\prime}}, \alpha_{j^{\prime}}\right)\right]\right)}{\sum_{j=1}^{n}(1+M)}\right) \\
& \leqslant \triangle\left(\frac{\sum_{j=1}^{n}(1+M) \triangle^{-1}\left(\max _{j}\left(\left[\left(s_{j}, \alpha_{j}\right),\left(s_{j^{\prime}}, \alpha_{j^{\prime}}\right)\right]\right)\right)}{\sum_{j=1}^{n}(1+M)}\right)
\end{aligned}
$$

that means

$$
\begin{aligned}
& \min _{j}\left(\left[\left(s_{j}, \alpha_{j}\right),\left(s_{j^{\prime}}, \alpha_{j^{\prime}}\right)\right]\right) \\
& \quad \leqslant \operatorname{LI} 2 \operatorname{TPA}\left(\left[\left(s_{1}, \alpha_{1}\right),\left(s_{1^{\prime}}, \alpha_{1^{\prime}}\right)\right], \ldots,\left[\left(s_{n}, \alpha_{n}\right),\left(s_{n^{\prime}}, \alpha_{n^{\prime}}\right)\right]\right) \\
& \quad \leqslant \max _{j}\left(\left[\left(s_{j}, \alpha_{j}\right),\left(s_{j^{\prime}}, \alpha_{j^{\prime}}\right)\right]\right)
\end{aligned}
$$

In Eq.(1), all the aggregated arguments are of equal importance. However, in many cases, the weights of the arguments should be taken into account. For example, in many group decision making problems, the importance degrees associated with decision makers should not be treated as equally important, thus, need to be assigned different weights. Suppose that each aggregated argument has a weight indicating its importance, then we define the weighted form of Eq.(1) as follows:

$$
\begin{aligned}
& \operatorname{LI2TWPA}\left(\left[\left(s_{1}, \alpha_{1}\right),\left(s_{1^{\prime}}, \alpha_{1^{\prime}}\right)\right], \ldots,\left[\left(s_{n}, \alpha_{n}\right),\left(s_{n^{\prime}}, \alpha_{n^{\prime}}\right)\right]\right) \\
& =\triangle\left(\frac{\sum_{j=1}^{n} w_{j}(1+M) \triangle^{-1}\left(\left[\left(s_{j}, \alpha_{j}\right),\left(s_{j^{\prime}}, \alpha_{j^{\prime}}\right)\right]\right)}{\sum_{j=1}^{n} w_{j}(1+M)}\right)
\end{aligned}
$$

with the condition $w_{j} \in[0,1], j=1,2, \ldots, n$, $\sum_{j=1}^{n} w_{j}=1$.

We call Eq.(2) a linguistic interval 2-tuple weighted power average(LI2TWPA) operator. Especially, if $W=\left(\frac{1}{n}, \frac{1}{n}, \ldots, \frac{1}{n}\right)^{T}$, then the LI2TWPA operator reduces to the LI2TPA operator.

Similarly, the linguistic interval 2-tuple weighted power average operator has the properties such as idempotency, boundedness, but commutativity property does not hold. In fact, if $\left\{\left[\left(s_{1}^{*}, \alpha_{1}^{*}\right),\left(s_{1^{\prime}}^{*}, \alpha_{1^{\prime}}^{*}\right)\right], \ldots,\left[\left(s_{n}^{*}, \alpha_{n}^{*}\right),\left(s_{n^{\prime}}^{*}, \alpha_{n^{\prime}}^{*}\right)\right]\right\}$ is any permutation of $\left\{\left[\left(s_{1}, \alpha_{1}\right),\left(s_{1^{\prime}}, \alpha_{1^{\prime}}\right)\right], \ldots,\left[\left(s_{n}, \alpha_{n}\right),\left(s_{n^{\prime}}\right.\right.\right.$, $\left.\left.\left.\alpha_{n^{\prime}}\right)\right]\right\}$, then

$$
\begin{aligned}
& \operatorname{LI2TWPA}\left(\left[\left(s_{1}, \alpha_{1}\right),\left(s_{1^{\prime}}, \alpha_{1^{\prime}}\right)\right], \ldots,\left[\left(s_{n}, \alpha_{n}\right),\left(s_{n^{\prime}}, \alpha_{n^{\prime}}\right)\right]\right) \\
& =\triangle\left(\frac{\sum_{j=1}^{n} w_{j}(1+M) \triangle^{-1}\left(\left[\left(s_{j}, \alpha_{j}\right),\left(s_{j^{\prime}}, \alpha_{j^{\prime}}\right)\right]\right)}{\sum_{j=1}^{n} w_{j}(1+M)}\right) \\
& \operatorname{LI} 2 T W P A\left(\left[\left(s_{1}^{*}, \alpha_{1}^{*}\right),\left(s_{1^{\prime}}^{*}, \alpha_{1^{\prime}}^{*}\right)\right], \ldots,\left[\left(s_{n}^{*}, \alpha_{n}^{*}\right),\left(s_{n^{\prime}}^{*}, \alpha_{n^{\prime}}^{*}\right)\right]\right) \\
& =\triangle\left(\frac{\sum_{j=1}^{n} w_{j}\left(1+M^{*}\right) \triangle^{-1}\left(\left[\left(s_{j}^{*}, \alpha_{j}^{*}\right),\left(s_{j^{\prime}}^{*}, \alpha_{j^{\prime}}^{*}\right)\right]\right)}{\sum_{j=1}^{n} w_{j}\left(1+M^{*}\right)}\right)
\end{aligned}
$$

where $\quad M=T\left(\left[\left(s_{j}^{*}, \alpha_{j}^{*}\right),\left(s_{j^{\prime}}^{*}, \alpha_{j^{\prime}}^{*}\right)\right]\right), \quad M^{*}=$ $T\left(\left[\left(s_{j}^{*}, \alpha_{j}^{*}\right),\left(s_{j^{\prime}}^{*}, \alpha_{j^{\prime}}^{*}\right)\right]\right)$, thus LI2TWPA $\left(\left[\left(s_{1}, \alpha_{1}\right),\left(s_{1^{\prime}}\right.\right.\right.$, $\left.\left.\left.\alpha_{1^{\prime}}\right)\right], \ldots,\left[\left(s_{n}, \alpha_{n}\right),\left(s_{n^{\prime}}, \alpha_{n^{\prime}}\right)\right]\right)=\operatorname{LI} 2 T W P A\left(\left[\left(s_{1}^{*}, \alpha_{1}^{*}\right)\right.\right.$, $\left.\left.\left(s_{1^{\prime}}^{*}, \alpha_{1^{\prime}}^{*}\right)\right], \ldots,\left[\left(s_{n}^{*}, \alpha_{n}^{*}\right),\left(s_{n^{\prime}}^{*}, \alpha_{n^{\prime}}^{*}\right)\right]\right)$ generally does not hold.

Theorem 5. Let Sup $\left(\left[\left(s_{i}, \alpha_{i}\right),\left(s_{i^{\prime}}, \alpha_{i^{\prime}}\right)\right],\left[\left(s_{j}, \alpha_{j}\right),\left(s_{j^{\prime}}\right.\right.\right.$, $\left.\left.\left.\alpha_{j^{\prime}}\right)\right]\right)=k$, for all $i \neq j$, then

$$
\begin{aligned}
& \operatorname{LI} 2 T W P A\left(\left[\left(s_{1}, \alpha_{1}\right),\left(s_{1^{\prime}}, \alpha_{1^{\prime}}\right)\right], \ldots,\left[\left(s_{n}, \alpha_{n}\right),\left(s_{n^{\prime}}, \alpha_{n^{\prime}}\right)\right]\right) \\
& =\triangle\left(\sum_{j=1}^{n} w_{j} \triangle^{-1}\left(\left[\left(s_{j}, \alpha_{j}\right),\left(s_{j^{\prime}}, \alpha_{j^{\prime}}\right)\right]\right)\right)
\end{aligned}
$$

which indicates that when all the supports are the same, the LI2TWPA operator is simply a linguistic interval 2-tuple weighted average(LI2TWA) operator.

Proof. If $\operatorname{Sup}\left(\left[\left(s_{i}, \alpha_{i}\right),\left(s_{i^{\prime}}, \alpha_{i^{\prime}}\right)\right],\left[\left(s_{j}, \alpha_{j}\right),\left(s_{j^{\prime}}, \alpha_{j^{\prime}}\right)\right]\right)=$ $k$, for all $i \neq j$, then

$$
T\left(\left[\left(s_{j}, \alpha_{j}\right),\left(s_{j^{\prime}}, \alpha_{j^{\prime}}\right)\right]\right)=(n-1) k
$$

thus

$$
\begin{aligned}
& \operatorname{LI2TWPA}\left(\left[\left(s_{1}, \alpha_{1}\right),\left(s_{1^{\prime}}, \alpha_{1^{\prime}}\right)\right], \ldots,\left[\left(s_{n}, \alpha_{n}\right),\left(s_{n^{\prime}}, \alpha_{n^{\prime}}\right)\right]\right) \\
& =\triangle\left(\frac{\sum_{j=1}^{n} w_{j}(1+(n-1) k) \triangle^{-1}\left(\left[\left(s_{j}, \alpha_{j}\right),\left(s_{j^{\prime}}, \alpha_{j^{\prime}}\right)\right]\right)}{\sum_{j=1}^{n} w_{j}(1+(n-1) k)}\right) \\
& =\triangle\left(\sum_{j=1}^{n} w_{j} \triangle^{-1}\left(\left[\left(s_{j}, \alpha_{j}\right),\left(s_{j^{\prime}}, \alpha_{j^{\prime}}\right)\right]\right)\right)
\end{aligned}
$$

which is simply a linguistic interval 2-tuple weighted average (LI2TWA) operator.

Base on the OWA operator ${ }^{25}$ and the LI2TPA operator, we define a linguistic interval 2-tuple power 
ordered weighted average (LI2TPOWA) operator as follows.

Definition 6. Let $\left\{\left[\left(s_{1}, \alpha_{1}\right),\left(s_{1^{\prime}}, \alpha_{1^{\prime}}\right)\right], \ldots,\left[\left(s_{n}, \alpha_{n}\right)\right.\right.$, $\left.\left.\left(s_{n^{\prime}}, \alpha_{n^{\prime}}\right)\right]\right\}$ be a collection of linguistic interval 2tuples, then a linguistic interval 2-tuple power ordered weighted average (LI2TPOWA) operator is defined as follows:

$\operatorname{LI2TPOWA}\left(\left[\left(s_{1}, \alpha_{1}\right),\left(s_{1^{\prime}}, \alpha_{1^{\prime}}\right)\right], \ldots,\left[\left(s_{n}, \alpha_{n}\right),\left(s_{n^{\prime}}, \alpha_{n^{\prime}}\right)\right]\right)$ $=\triangle\left(\sum_{j=1}^{n} u_{j} \triangle^{-1}\left(\left[\left(s_{\sigma(j)}, \alpha_{\sigma(j)}\right),\left(s_{\sigma(j)^{\prime}}, \alpha_{\sigma(j)^{\prime}}\right)\right]\right)\right)$

where $(\sigma(1), \sigma(2), \ldots, \sigma(n))$ is a permutation of $(1,2, \ldots, n)$ such that $\left[\left(s_{\sigma(j-1)}, \alpha_{\sigma(j-1)}\right),\left(s_{\sigma(j-1)^{\prime}}\right.\right.$, $\left.\left.\alpha_{\sigma(j-1)^{\prime}}\right)\right] \geqslant\left[\left(s_{\sigma(j)}, \alpha_{\sigma(j)}\right),\left(s_{\sigma(j)^{\prime}}, \alpha_{\sigma(j)^{\prime}}\right)\right]$ for all $j$, and

$$
\begin{gathered}
u_{j}=g\left(\frac{B_{j}}{T V}\right)-g\left(\frac{B_{j-1}}{T V}\right), \\
B_{j}=\sum_{i=1}^{j} V_{\sigma(i)}, T V=\sum_{j=1}^{n} V_{\sigma(j)}, \\
V_{\sigma(j)}=1+T\left(\left[\left(s_{\sigma(j)}, \alpha_{\sigma(j)}\right),\left(s_{\sigma(j)^{\prime}}, \alpha_{\sigma(j)^{\prime}}\right)\right]\right)
\end{gathered}
$$

and $g:[0,1] \rightarrow[0,1]$ is a basic unit-interval monotonic(BUM) function having the following properties: (1) $g(0)=0$; (2) $g(1)=1$; (3) $g(x) \geqslant g(y)$, if $x>y . T\left(\left[\left(s_{\sigma(j)}, \alpha_{\sigma(j)}\right),\left(s_{\sigma(j)^{\prime}}, \alpha_{\sigma(j)^{\prime}}\right)\right]\right)$ denotes the support of the $j$ th largest argument by all the other arguments, i.e., $T\left(\left[\left(s_{\sigma(j)}, \alpha_{\sigma(j)}\right),\left(s_{\sigma(j)^{\prime}}, \alpha_{\sigma(j)^{\prime}}\right)\right]\right)=$ $\sum_{i=1, i \neq j}^{n} \operatorname{Sup}\left(\left[\left(s_{\sigma(j)}, \alpha_{\sigma(j)}\right),\left(s_{\sigma(j)^{\prime}}, \alpha_{\sigma(j)^{\prime}}\right)\right],\left[\left(s_{\sigma(i)}\right.\right.\right.$, $\left.\left.\left.\alpha_{\sigma(i)}\right),\left(s_{\sigma(i)^{\prime}}, \alpha_{\sigma(i)^{\prime}}\right)\right]\right)$, and $\operatorname{Sup}\left(\left[\left(s_{\sigma(j)}, \alpha_{\sigma(j)}\right),\left(s_{\sigma(j)^{\prime}}\right.\right.\right.$, $\left.\left.\left.\alpha_{\sigma(j)^{\prime}}\right)\right],\left[\left(s_{\sigma(i)}, \alpha_{\sigma(i)}\right),\left(s_{\sigma(i)^{\prime}}, \alpha_{\sigma(i)^{\prime}}\right)\right]\right)$ is the support for the $j$ th largest argument from the $i$ th largest argument.

Especially, if $g(x)=x$, then the LI2TPOWA operator reduces to the LI2TPA operator. Similar to the LI2TPA operator, we have the following results.

Theorem 6. Let $\operatorname{Sup}\left(\left[\left(s_{\sigma(i)}, \alpha_{\sigma(i)}\right),\left(s_{\sigma(i)^{\prime}}, \alpha_{\sigma(i)^{\prime}}\right)\right]\right.$, $\left.\left[\left(s_{\sigma(j)}, \alpha_{\sigma(j)}\right),\left(s_{\sigma(j)^{\prime}}, \alpha_{\sigma(j)^{\prime}}\right)\right]\right)=k$, for all $i \neq j$, and $g(x)=x$, then LI2TPOWA $\left(\left[\left(s_{1}, \alpha_{1}\right),\left(s_{1^{\prime}}, \alpha_{1^{\prime}}\right)\right], \ldots\right.$, $\left.\left[\left(s_{n}, \alpha_{n}\right),\left(s_{n^{\prime}}, \alpha_{n^{\prime}}\right)\right]\right)=\triangle\left(\frac{1}{n} \sum_{j=1}^{n} \triangle^{-1}\left(\left[\left(s_{j}, \alpha_{j}\right),\left(s_{j^{\prime}}\right.\right.\right.\right.$, $\left.\left.\left.\left.\alpha_{j^{\prime}}\right)\right]\right)\right)$, which indicates that when all the supports are the same, the LI2TPOWA reduces to the simple linguistic interval 2-tuple average operator.

Theorem 7. (Commutativity) Let $\left\{\left[\left(s_{1}, \alpha_{1}\right),\left(s_{1^{\prime}}, \alpha_{1^{\prime}}\right)\right]\right.$, $\left.\ldots,\left[\left(s_{n}, \alpha_{n}\right),\left(s_{n^{\prime}}, \alpha_{n^{\prime}}\right)\right]\right\}$ and $\left\{\left[\left(s_{1}^{*}, \alpha_{1}^{*}\right),\left(s_{1^{\prime}}^{*}, \alpha_{1^{\prime}}^{*}\right)\right], \ldots\right.$, $\left.\left[\left(s_{n}^{*}, \alpha_{n}^{*}\right),\left(s_{n^{\prime}}^{*}, \alpha_{n^{\prime}}^{*}\right)\right]\right\}$ be two collections of linguistic interval 2-tuples, then

$$
\begin{aligned}
& \operatorname{LI2TPOWA}\left(\left[\left(s_{1}, \alpha_{1}\right),\left(s_{1^{\prime}}, \alpha_{1^{\prime}}\right)\right], \ldots,\left[\left(s_{n}, \alpha_{n}\right),\left(s_{n^{\prime}}, \alpha_{n^{\prime}}\right)\right]\right) \\
& =\operatorname{LI} 2 \mathrm{TPOWA}\left(\left[\left(s_{1}^{*}, \alpha_{1}^{*}\right),\left(s_{1^{\prime}}^{*}, \alpha_{1^{\prime}}^{*}\right)\right], \ldots,\left[\left(s_{n}^{*}, \alpha_{n}^{*}\right),\left(s_{n^{\prime}}^{*}, \alpha_{n^{\prime}}^{*}\right)\right]\right)
\end{aligned}
$$

where $\left\{\left[\left(s_{1}^{*}, \alpha_{1}^{*}\right),\left(s_{1^{\prime}}^{*}, \alpha_{1^{\prime}}^{*}\right)\right], \ldots,\left[\left(s_{n}^{*}, \alpha_{n}^{*}\right),\left(s_{n^{\prime}}^{*}, \alpha_{n^{\prime}}^{*}\right)\right]\right\}$ is any permutation of $\left\{\left[\left(s_{1}, \alpha_{1}\right),\left(s_{1^{\prime}}, \alpha_{1^{\prime}}\right)\right], \ldots,\left[\left(s_{n}, \alpha_{n}\right)\right.\right.$, $\left.\left.\left(s_{n^{\prime}}, \alpha_{n^{\prime}}\right)\right]\right\}$.

Theorem 8. (Idempotency) Let $\left\{\left[\left(s_{1}, \alpha_{1}\right),\left(s_{1^{\prime}}, \alpha_{1^{\prime}}\right)\right]\right.$, $\left.\ldots,\left[\left(s_{n}, \alpha_{n}\right),\left(s_{n^{\prime}}, \alpha_{n^{\prime}}\right)\right]\right\}$ be a collection of linguistic interval 2-tuples, if all $\left[\left(s_{j}, \alpha_{j}\right),\left(s_{j^{\prime}}, \alpha_{j^{\prime}}\right)\right](j=$ $1,2, \ldots, n)$ are equal, i.e., $\left[\left(s_{j}, \alpha_{j}\right),\left(s_{j^{\prime}}, \alpha_{j^{\prime}}\right)\right]=$ $\left[(s, \alpha),\left(s^{\prime}, \alpha^{\prime}\right)\right]$, for all $j$, then LI2T POWA $\left(\left[\left(s_{1}, \alpha_{1}\right)\right.\right.$, $\left.\left.\left(s_{1^{\prime}}, \alpha_{1^{\prime}}\right)\right], \ldots,\left[\left(s_{n}, \alpha_{n}\right),\left(s_{n^{\prime}}, \alpha_{n^{\prime}}\right)\right]\right)=\left[(s, \alpha),\left(s^{\prime}, \alpha^{\prime}\right)\right]$.

Theorem 9. (Boundedness) Let $\left\{\left[\left(s_{1}, \alpha_{1}\right),\left(s_{1^{\prime}}, \alpha_{1^{\prime}}\right)\right]\right.$, $\left.\ldots,\left[\left(s_{n}, \alpha_{n}\right),\left(s_{n^{\prime}}, \alpha_{n^{\prime}}\right)\right]\right\}$ be a collection of linguistic interval 2-tuples, then $\min _{j}\left(\left[\left(s_{j}, \alpha_{j}\right),\left(s_{j^{\prime}}, \alpha_{j^{\prime}}\right)\right]\right) \leqslant$ LI2TPOWA $\left(\left[\left(s_{1}, \alpha_{1}\right),\left(s_{1^{\prime}}, \alpha_{1^{\prime}}\right)\right], \ldots,\left[\left(s_{n}, \alpha_{n}\right),\left(s_{n^{\prime}}\right.\right.\right.$, $\left.\left.\left.\alpha_{n^{\prime}}\right)\right]\right) \leqslant \max _{j}\left(\left[\left(s_{j}, \alpha_{j}\right),\left(s_{j^{\prime}}, \alpha_{j^{\prime}}\right)\right]\right)$.

According to above theoretical analysis, we know that both the LI2TWPA and LI2TPOWA operators can take into account the aggregated arguments and their relationships. Then, the difference between the two new operators is that the LI2TWPA operator emphasizes the importance of each aggregated argument, while the LI2TPOWA operator more weights the importance of the ordered position of each aggregated argument. In many multiple attribute group decision making problems, some individuals may provide unduly high or unduly low performance values to their preferred or repugnant objects. The prominent advantage of the developed operators is that the associated weights are derived directly from the aggregated performance values by using the support measure. The more support the performance value provided by a decision maker to all the other aggregated performance values provided by other decision makers, the more the associated weight. Thus, the new operators can reduce the influence of 
these unduly high or unduly low performance values, thereby making the decision making process more reasonable.

\section{Approaches to multiple attribute group decision making based on linguistic interval 2-tuple power aggregation operators}

In this section, we apply the above linguistic interval 2-tuple power aggregation operators to solve multiple attribute group decision making under linguistic environment.

For a multiple attribute group decision making problem under linguistic environment, let $X=$ $\left\{x_{1}, x_{2}, \ldots, x_{m}\right\}$ be a set of finite alternatives, $C=$ $\left\{c_{1}, c_{2}, \ldots, c_{n}\right\}$ be a set of finite attributes and $\omega=$ $\left(\omega_{1}, \omega_{2}, \ldots, \omega_{n}\right)^{T}$ be the weighting vector of attributes, where $\omega_{j} \in[0,1], j=1,2, \ldots, n, \sum_{j=1}^{n} \omega_{j}=$ 1. Let $D=\left\{d_{1}, d_{2}, \ldots, d_{t}\right\}$ be the set of decision makers, whose weighting vector is $w=$ $\left(w_{1}, w_{2}, \ldots, w_{t}\right)^{T}$, with $w_{k} \in[0,1]$ and $\sum_{k=1}^{t} w_{k}=1$. Assume that each decision maker provides his/her own decision matrix $A^{(k)}=\left(a_{i j}^{k}\right)_{m \times n}$, in which $a_{i j}^{k}$ is a performance value provided by the decision maker $d_{k} \in D$, for the alternative $x_{i} \in X$ with respect to the attribute $c_{j} \in C$ and $a_{i j}^{k}$ takes the form of interval, denote by $\left[s_{i j}^{l(k)}, s_{i j}^{r(k)}\right]$, with $s_{i j}^{l(k)}, s_{i j}^{r(k)} \in S$, $S=\left\{s_{0}, s_{1}, \ldots, s_{g}\right\}$ and $s_{i j}^{l(k)} \leqslant s_{i j}^{r(k)}$.

Then, we apply the LI2TWPA operator to develop an approach to multiple attribute group decision making under linguistic environment, which can be summarized as follows:

\subsection{Approach I}

Step 1. Transform the linguistic interval decision matrix $A^{(k)}=\left(a_{i j}^{k}\right)_{m \times n}$, i.e., $A^{(k)}=\left(\left[s_{i j}^{l(k)}, s_{i j}^{r(k)}\right]\right)_{m \times n}$ into linguistic interval 2-tuple decision matrix $\bar{A}^{(k)}=$ $\left(\left[\left(s_{i j}^{l(k)}, 0\right),\left(s_{i j}^{r(k)}, 0\right)\right]\right)_{m \times n}$.

Step 2. Calculate the support measure for the linguistic interval 2-tuple $\left[\left(s_{i j}^{l(k)}, 0\right),\left(s_{i j}^{r(k)}, 0\right)\right]$ from $\left[\left(s_{i j}^{l(h)}, 0\right),\left(s_{i j}^{r(h)}, 0\right)\right]$, i.e., $\operatorname{Sup}\left(\left[\left(s_{i j}^{l(k)}, 0\right),\left(s_{i j}^{r(k)}, 0\right)\right],\left[\left(s_{i j}^{l(h)}, 0\right),\left(s_{i j}^{r(h)}, 0\right)\right]\right)=1-$ $d\left(\left[\left(s_{i j}^{l(k)}, 0\right),\left(s_{i j}^{r(k)}, 0\right)\right],\left[\left(s_{i j}^{l(h)}, 0\right),\left(s_{i j}^{r(h)}, 0\right)\right]\right), \quad h=$
$1,2, \ldots, t, i=1,2, \ldots, m, j=1,2, \ldots, n$, and $k \neq h$, which satisfy the support conditions (1)-(3) in section 3, without loss of generality, here we let

$$
\begin{aligned}
& d\left(\left[\left(s_{i j}^{l(k)}, 0\right),\left(s_{i j}^{r(k)}, 0\right)\right],\left[\left(s_{i j}^{l(h)}, 0\right),\left(s_{i j}^{r(h)}, 0\right)\right]\right) \\
& =\frac{1}{g} \sqrt{\frac{1}{2}(M+N)}
\end{aligned}
$$

where $M=\left(\triangle^{-1}\left(s_{i j}^{l(h)}, 0\right)-\triangle^{-1}\left(s_{i j}^{l(k)}, 0\right)\right)^{2}, N=$ $\left(\triangle^{-1}\left(s_{i j}^{r(h)}, 0\right)-\triangle^{-1}\left(s_{i j}^{r(k)}, 0\right)\right)^{2}$.

Step 3. Calculate the support $T\left(\left[\left(s_{i j}^{l(k)}, 0\right),\left(s_{i j}^{r(k)}, 0\right)\right]\right)$ of the linguistic interval 2-tuple $\left[\left(s_{i j}^{l(k)}, 0\right),\left(s_{i j}^{r(k)}, 0\right)\right]$ by other linguistic interval 2-tuples $\left[\left(s_{i j}^{l(h)}, 0\right),\left(s_{i j}^{r(h)}, 0\right)\right]$ $(h=1,2, \ldots, t$ and $k \neq h)$,

$$
\begin{aligned}
& T\left(\left[\left(s_{i j}^{l(k)}, 0\right),\left(s_{i j}^{r(k)}, 0\right)\right]\right) \\
& =\sum_{\substack{h=1 \\
h \neq k}}^{t} \operatorname{Sup}\left(\left[\left(s_{i j}^{l(k)}, 0\right),\left(s_{i j}^{r(k)}, 0\right)\right],\left[\left(s_{i j}^{l(h)}, 0\right),\left(s_{i j}^{r(h)}, 0\right)\right]\right)
\end{aligned}
$$

and then utilize the weights $w_{k}(k=1,2, \ldots, t)$ of the decision makers $d_{k}(k=1,2, \ldots, t)$ to calculate the weights $\varpi_{i j}^{(k)}(k=1,2, \ldots, t)$ associated with the linguistic interval 2-tuples $\left[\left(s_{i j}^{l(k)}, 0\right),\left(s_{i j}^{r(k)}, 0\right)\right](k=$ $1,2, \ldots, t)$,

$$
\varpi_{i j}^{(k)}=\frac{w_{k}\left(1+T\left(\left[\left(s_{i j}^{l(k)}, 0\right),\left(s_{i j}^{r(k)}, 0\right)\right]\right)\right)}{\sum_{k=1}^{t} w_{k}\left(1+T\left(\left[\left(s_{i j}^{l(k)}, 0\right),\left(s_{i j}^{r(k)}, 0\right)\right]\right)\right)}
$$

where ${\varpi_{i j}^{(k)}}_{0} \geqslant 0, k=1,2, \ldots, t$, and $\sum_{k=1}^{t} \varpi_{i j}^{(k)}=1$.

Step 4. Utilize the LI2TWPA operator to aggregate all the individual decision matrixes $\bar{A}^{(k)}=\left(\left[\left(s_{i j}^{l(k)}, 0\right),\left(s_{i j}^{r(k)}, 0\right)\right]\right)_{m \times n}$ into the collective decision matrix $\bar{A}=$ $\left(\bar{a}_{i j}\right)_{m \times n}=\left(\left[\left(s_{i j}^{l}, \alpha_{i j}^{l}\right),\left(s_{i j}^{r}, \alpha_{i j}^{r}\right)\right]\right)_{m \times n}$, where $\bar{a}_{i j}=$ LI2TWPA $\left(\left[\left(s_{i j}^{l(1)}, 0\right),\left(s_{i j}^{r(1)}, 0\right)\right], \ldots,\left[\left(s_{i j}^{l(t)}, 0\right),\left(s_{i j}^{r(t)}\right.\right.\right.$, $0)])=\triangle\left(\sum_{k=1}^{t} \varpi_{i j}^{(k)} \triangle^{-1}\left(\left[\left(s_{i j}^{l(k)}, 0\right),\left(s_{i j}^{r(k)}, 0\right)\right]\right)\right)$.

Step 5. Utilize the decision matrix $\bar{A}=$ $\left(\left[\left(s_{i j}^{l}, \alpha_{i j}^{l}\right),\left(s_{i j}^{r}, \alpha_{i j}^{r}\right)\right]\right)_{m \times n}$ and the LI2TWA operator, i.e., $\bar{z}_{i}=L I 2 T W A_{\omega}\left(\left[\left(s_{i 1}^{l}, \alpha_{i 1}^{l}\right),\left(s_{i 1}^{r}, \alpha_{i 1}^{r}\right)\right], \ldots,\left[\left(s_{i n}^{l}, \alpha_{i n}^{l}\right)\right.\right.$, $\left.\left.\left(s_{i n}^{r}, \alpha_{i n}^{r}\right)\right]\right)=\triangle\left(\sum_{j=1}^{n} \omega_{j} \triangle^{-1}\left(\left[\left(s_{i j}^{l}, \alpha_{i j}^{l}\right),\left(s_{i j}^{r}, \alpha_{i j}^{r}\right)\right]\right)\right)$ to derive the collective overall performance values $\bar{z}_{i}(i=1,2, \ldots, m)$ of the alternatives $x_{i}(i=$ 
$1,2, \ldots, m)$, where $\omega=\left(\omega_{1}, \omega_{2}, \ldots, \omega_{n}\right)^{T}$ is the weighting vector of attributes.

Step 6. Rank all the alternatives and select the best one(s) in accordance with the overall values of linguistic interval 2-tuples $\bar{z}_{i}(i=1,2, \ldots, m)$.

Step 7. End.

If the information about the weights of decision makers are unknown, we utilize the LI2TPOWA operator to develop another approach to multiple attribute group decision making under linguistic environment, which involves the following steps:

\subsection{Approach II}

Step 1. For this step, see Approach I.

Step 2. Calculate $\operatorname{Sup}\left(\left[\left(s_{i j}^{l(\sigma(k))}, 0\right),\left(s_{i j}^{r(\sigma(k))}, 0\right)\right]\right.$, $\left.\left[\left(s_{i j}^{l(\sigma(h))}, 0\right),\left(s_{i j}^{r(\sigma(h))}, 0\right)\right]\right) \quad(h=1,2, \ldots, t, \quad i=$ $1,2, \ldots, m, j=1,2, \ldots, n$ and $k \neq h)$ according to Step 2 of approach I, which indicates the support for the $k$ th largest linguistic interval 2-tuple $\left[\left(s_{i j}^{l(\sigma(k))}, 0\right),\left(s_{i j}^{r(\sigma(k))}, 0\right)\right]$ from the $h$ th largest linguistic interval 2-tuple $\left[\left(s_{i j}^{l(\sigma(h))}, 0\right),\left(s_{i j}^{r(\sigma(h))}, 0\right)\right]$.

Step 3. Calculate the support $T\left(\left[\left(s_{i j}^{l(\sigma(k))}, 0\right)\right.\right.$, $\left.\left.\left(s_{i j}^{r(\sigma(k))}, 0\right)\right]\right)$ of the $k$ th largest linguistic interval 2-tuple $\left[\left(s_{i j}^{l(\sigma(k))}, 0\right),\left(s_{i j}^{r(\sigma(k))}, 0\right)\right]$ by the other linguistic interval 2-tuples $\left[\left(s_{i j}^{l(q)}, 0\right),\left(s_{i j}^{r(q)}, 0\right)\right] \quad(q=1,2, \ldots, t \quad$ and $\quad q \neq$ $\sigma(k))$, i.e., $\quad T\left(\left[\left(s_{i j}^{l(\sigma(k))}, 0\right),\left(s_{i j}^{r(\sigma(k))}, 0\right)\right]\right)=$ $\sum_{h=1, h \neq k}^{t} \operatorname{Sup}\left(\left[\left(s_{i j}^{l(\sigma(k))}, 0\right),\left(s_{i j}^{r(\sigma(k))}, 0\right)\right],\left[\left(s_{i j}^{l(\sigma(h))}, 0\right)\right.\right.$, $\left.\left.\left(s_{i j}^{r(\sigma(h))}, 0\right)\right]\right)$, and utilize Eq.(3), Eq.(4) and Eq.(5) to calculate the weight $u_{i j}^{(k)}$ associated with the $k$ th largest linguistic interval 2-tuple $\left[\left(s_{i j}^{l(\sigma(k))}, 0\right),\left(s_{i j}^{r(\sigma(k))}, 0\right)\right]$, where

$$
\begin{gathered}
u_{i j}^{(k)}=g\left(\frac{B_{i j}^{(k)}}{T V_{i j}}\right)-g\left(\frac{B_{i j}^{(k-1)}}{T V_{i j}}\right), \\
B_{i j}^{(k)}=\sum_{q=1}^{k} V_{i j}^{\sigma(q)}, T V_{i j}=\sum_{q=1}^{t} V_{i j}^{\sigma(q)}, \\
V_{i j}^{\sigma(q)}=1+T\left(\left[\left(s_{i j}^{l(\sigma(q))}, 0\right),\left(s_{i j}^{r(\sigma(q))}, 0\right)\right]\right),
\end{gathered}
$$

and $u_{i j}^{(k)} \geqslant 0, k=1,2, \ldots, t$, and $\sum_{k=1}^{t} u_{i j}^{(k)}=1, g$ is the BUM function, as described in section 3 .

Step 4. Utilize the LI2TPOWA operator to aggregate all the individual decision matrixes $\bar{A}^{(k)}=\left(\left[\left(s_{i j}^{l(k)}, 0\right),\left(s_{i j}^{r(k)}, 0\right)\right]\right)_{m \times n}$ into the collective decision matrix $\bar{A}=$ $\left(\bar{a}_{i j}\right)_{m \times n}=\left(\left[\left(s_{i j}^{l}, \alpha_{i j}^{l}\right),\left(s_{i j}^{r}, \alpha_{i j}^{r}\right)\right]\right)_{m \times n}$, where $\bar{a}_{i j}=$ LI2TPOWA $\left(\left[\left(s_{i j}^{l(1)}, 0\right),\left(s_{i j}^{r(1)}, 0\right)\right], \ldots,\left[\left(s_{i j}^{l(t)}, 0\right),\left(s_{i j}^{r(t)}\right.\right.\right.$, $0)])=\triangle\left(\sum_{k=1}^{t} u_{i j}^{(k)} \triangle^{-1}\left(\left[\left(s_{i j}^{l(\sigma(k))}, 0\right),\left(s_{i j}^{r(\sigma(k))}, 0\right)\right]\right)\right)$.

Step 5. For this step, see Approach I.

Step 6. For this step, see Approach I.

Step 7. End.

\section{Illustrative example}

Example 1. Suppose an investment company wants to invest a sum of money in the best option. There is a panel with four possible alternatives of where to invest the money: $B_{1}$ is a car industry; $B_{2}$ is a food company; $B_{3}$ is a computer company; $B_{4}$ is an arms industry. The investment company must make a decision according to four attributes: $C_{1}$ is the risk analysis; $C_{2}$ is the growth analysis; $C_{3}$ is the social-political impact analysis. The weighting vector of attributes is $w=(0.3,0.5,0.2)$. Three experts, whose weighting vector is $\lambda=(0.3,0.3,0.4)$, are invited to provide their assessments for each alternative on each attribute using the predefined linguistic term set $S=\left\{s_{0}=\right.$ very poor, $s_{1}=$ poor, $s_{2}=$ slightly poor, $s_{3}=$ fair, $s_{4}=$ slightly good, $s_{5}=$ good, $s_{6}=$ very good $\}$.

The decision matrixes $A^{(k)}=\left(\left[s_{i j}^{l(k)}, s_{i j}^{r(k)}\right]\right)_{4 \times 3}(k=$ $1,2,3)$ are provided as follows:

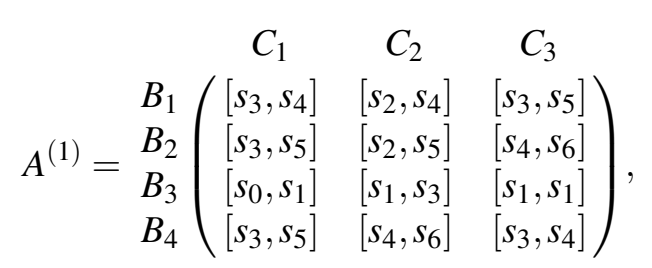




$$
\begin{aligned}
& \begin{array}{lll}
C_{1} & C_{2} & C_{3}
\end{array}
\end{aligned}
$$

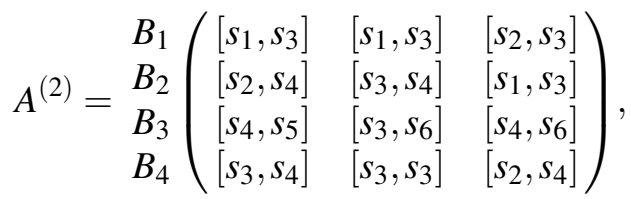

$$
\begin{aligned}
& \begin{array}{lll}
C_{1} & C_{2} & C_{3}
\end{array}
\end{aligned}
$$

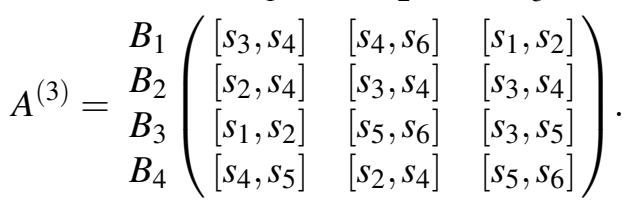

Consider that the weighting vector of the three decision makers are known, here, we utilize Approach I to reach the decision results:

Step 1. Transform the linguistic interval decision matrixes $A^{(k)}=\left(\left[s_{i j}^{l(k)}, s_{i j}^{r(k)}\right]\right)_{4 \times 3} \quad(k=$ $1,2,3)$ into linguistic interval 2-tuple decision matrixes $\bar{A}^{(k)}=\left(\left[\left(s_{i j}^{l(k)}, 0\right),\left(s_{i j}^{r(k)}, 0\right)\right]\right)_{4 \times 3}(k=1,2,3)$, $\bar{A}^{(1)}, \bar{A}^{(2)}$ and $\bar{A}^{(3)}$ are respectively listed in the following:

$$
\left(\begin{array}{lll}
{\left[\left(s_{3}, 0\right),\left(s_{4}, 0\right)\right]} & {\left[\left(s_{2}, 0\right),\left(s_{4}, 0\right)\right]} & {\left[\left(s_{3}, 0\right),\left(s_{5}, 0\right)\right]} \\
{\left[\left(s_{3}, 0\right),\left(s_{5}, 0\right)\right]} & {\left[\left(s_{2}, 0\right),\left(s_{5}, 0\right)\right]} & {\left[\left(s_{4}, 0\right),\left(s_{6}, 0\right)\right]} \\
{\left[\left(s_{0}, 0\right),\left(s_{1}, 0\right)\right]} & {\left[\left(s_{1}, 0\right),\left(s_{3}, 0\right)\right]} & {\left[\left(s_{1}, 0\right),\left(s_{1}, 0\right)\right]} \\
{\left[\left(s_{3}, 0\right),\left(s_{5}, 0\right)\right]} & {\left[\left(s_{4}, 0\right),\left(s_{6}, 0\right)\right]} & {\left[\left(s_{3}, 0\right),\left(s_{4}, 0\right)\right]}
\end{array}\right)
$$$$
\left(\begin{array}{lll}
{\left[\left(s_{1}, 0\right),\left(s_{3}, 0\right)\right]} & {\left[\left(s_{1}, 0\right),\left(s_{3}, 0\right)\right]} & {\left[\left(s_{2}, 0\right),\left(s_{3}, 0\right)\right]} \\
{\left[\left(s_{2}, 0\right),\left(s_{4}, 0\right)\right]} & {\left[\left(s_{3}, 0\right),\left(s_{4}, 0\right)\right]} & {\left[\left(s_{1}, 0\right),\left(s_{3}, 0\right)\right]} \\
{\left[\left(s_{4}, 0\right),\left(s_{5}, 0\right)\right]} & {\left[\left(s_{3}, 0\right),\left(s_{6}, 0\right)\right]} & {\left[\left(s_{4}, 0\right),\left(s_{6}, 0\right)\right]} \\
{\left[\left(s_{3}, 0\right),\left(s_{4}, 0\right)\right]} & {\left[\left(s_{3}, 0\right),\left(s_{3}, 0\right)\right]} & {\left[\left(s_{2}, 0\right),\left(s_{4}, 0\right)\right]}
\end{array}\right),
$$$$
\left(\begin{array}{lll}
{\left[\left(s_{3}, 0\right),\left(s_{4}, 0\right)\right]} & {\left[\left(s_{4}, 0\right),\left(s_{6}, 0\right)\right]} & {\left[\left(s_{1}, 0\right),\left(s_{2}, 0\right)\right]} \\
{\left[\left(s_{2}, 0\right),\left(s_{4}, 0\right)\right]} & {\left[\left(s_{3}, 0\right),\left(s_{4}, 0\right)\right]} & {\left[\left(s_{3}, 0\right),\left(s_{4}, 0\right)\right]} \\
{\left[\left(s_{1}, 0\right),\left(s_{2}, 0\right)\right]} & {\left[\left(s_{5}, 0\right),\left(s_{6}, 0\right)\right]} & {\left[\left(s_{3}, 0\right),\left(s_{5}, 0\right)\right]} \\
{\left[\left(s_{4}, 0\right),\left(s_{5}, 0\right)\right]} & {\left[\left(s_{2}, 0\right),\left(s_{4}, 0\right)\right]} & {\left[\left(s_{5}, 0\right),\left(s_{6}, 0\right)\right]}
\end{array}\right) .
$$

Step 2. According to Step 2 of Approach I, calculate the supports $\operatorname{Sup}\left(\left[\left(s_{i j}^{l(k)}, 0\right),\left(s_{i j}^{r(k)}, 0\right)\right],\left[\left(s_{i j}^{l(h)}, 0\right)\right.\right.$, $\left.\left.\left(s_{i j}^{r(h)}, 0\right)\right]\right) \quad(i=1,2,3,4, \quad j=1,2,3, \quad k, h=$ $1,2,3$ and $k \neq h)$. For simplicity, we denote $\left(\operatorname{Sup}\left(\left[\left(s_{i j}^{l(k)}, 0\right),\left(s_{i j}^{r(k)}, 0\right)\right],\left[\left(s_{i j}^{l(h)}, 0\right),\left(s_{i j}^{r(h)}, 0\right)\right]\right)\right)_{4 \times 3}$ by $S u p^{k h}$, which means the supports between $A^{(k)}$ and $A^{(h)}$, and they are listed in the following:

$$
\begin{aligned}
& \text { Sup }^{12}=\text { Sup }^{21}=\left(\begin{array}{lll}
0.7365 & 0.8333 & 0.7365 \\
0.8333 & 0.8333 & 0.5000 \\
0.3333 & 0.5751 & 0.3128 \\
0.8821 & 0.6273 & 0.8821
\end{array}\right), \\
& \text { Sup }^{13}=\text { Sup }^{31}=\left(\begin{array}{ccc}
1.000 & 0.6667 & 0.5751 \\
0.8333 & 0.8333 & 0.7365 \\
0.8333 & 0.4107 & 0.4730 \\
0.8821 & 0.6667 & 0.6667
\end{array}\right), \\
& \text { Sup }^{23}=\text { Sup }^{32}=\left(\begin{array}{ccc}
0.7365 & 0.5000 & 0.8333 \\
1.000 & 1.000 & 0.7365 \\
0.5000 & 0.7643 & 0.8333 \\
0.8333 & 0.8333 & 0.5751
\end{array}\right) .
\end{aligned}
$$

Step 3. Calculate the support $T\left(\left[\left(s_{i j}^{l(k)}, 0\right),\left(s_{i j}^{r(k)}, 0\right)\right]\right)$ of the linguistic interval 2-tuple $\left[\left(s_{i j}^{l(k)}, 0\right),\left(s_{i j}^{r(k)}, 0\right)\right]$ by other linguistic interval 2-tuples $\left[\left(s_{i j}^{l(h)}, 0\right),\left(s_{i j}^{r(h)}, 0\right)\right]$ $(h=1,2,3$, and $k \neq h)$, we denote $\left(T\left(\left[\left(s_{i j}^{l(k)}, 0\right),\left(s_{i j}^{r(k)}, 0\right)\right]\right)\right)_{4 \times 3}$ by $T_{k} \quad(k=1,2,3)$, which are shown in the following:

$$
\begin{aligned}
T_{1} & =\left(\begin{array}{lll}
1.7365 & 1.5000 & 1.3116 \\
1.6666 & 1.6666 & 1.2365 \\
1.1666 & 0.9858 & 0.7858 \\
1.7642 & 1.2940 & 1.5488
\end{array}\right), \\
T_{2} & =\left(\begin{array}{lll}
1.4730 & 1.3333 & 1.5698 \\
1.8333 & 1.8333 & 1.2365 \\
0.8333 & 1.3394 & 1.1461 \\
1.7154 & 1.4606 & 1.4572
\end{array}\right), \\
T_{3} & =\left(\begin{array}{lll}
1.7365 & 1.1667 & 1.4084 \\
1.8333 & 1.8333 & 1.4730 \\
1.3333 & 1.1750 & 1.3063 \\
1.7154 & 1.5000 & 1.2418
\end{array}\right) .
\end{aligned}
$$

Utilize the weights $\lambda=(0.3,0.3,0.4)$ of the three experts to calculate the weights $\varpi_{i j}^{(k)}(i=1,2,3,4, \quad j=1,2,3$, and $k=1,2,3)$ associated with the linguistic interval 2-tuples $\left[\left(s_{i j}^{l(k)}, 0\right),\left(s_{i j}^{r(k)}, 0\right)\right](i=1,2,3,4, j=1,2,3$, and $k=$ 
$1,2,3)$, we denote $\left(\varpi_{i j}^{(k)}\right)_{4 \times 3}$ by $R_{k}$, which are in the following:

$$
\begin{aligned}
& R_{1}=\left(\begin{array}{lll}
0.3089 & 0.3237 & 0.2856 \\
0.2874 & 0.2874 & 0.2878 \\
0.3047 & 0.2748 & 0.2549 \\
0.3038 & 0.2836 & 0.3188
\end{array}\right), \\
& R_{2}=\left(\begin{array}{lll}
0.2792 & 0.3022 & 0.3175 \\
0.3054 & 0.3054 & 0.2878 \\
0.2578 & 0.3238 & 0.3063 \\
0.2984 & 0.3042 & 0.3073
\end{array}\right), \\
& R_{3}=\left(\begin{array}{lll}
0.4119 & 0.3741 & 0.3968 \\
0.4072 & 0.4072 & 0.4243 \\
0.4375 & 0.4014 & 0.4389 \\
0.3979 & 0.4121 & 0.3739
\end{array}\right) .
\end{aligned}
$$

Step 4. Utilize the LI2TWPA operator to aggregate all the individual decision matrixes $\bar{A}^{(k)}=\left(\left[\left(s_{i j}^{l(k)}, 0\right),\left(s_{i j}^{r(k)}, 0\right)\right]\right)_{4 \times 3} \quad(k=1,2,3)$ into the collective decision matrix $\bar{A}=\left(\bar{a}_{i j}\right)_{4 \times 3}=$ $\left(\left[\left(s_{i j}^{l}, \alpha_{i j}^{l}\right),\left(s_{i j}^{r}, \alpha_{i j}^{r}\right)\right]\right)_{4 \times 3}$, which is shown in Eq.(6).

Step 5. Utilize the decision matrix $\bar{A}=$ $\left(\left[\left(s_{i j}^{l}, \alpha_{i j}^{l}\right),\left(s_{i j}^{r}, \alpha_{i j}^{r}\right)\right]\right)_{4 \times 3}$ and the LI2TWA operator to derive the collective overall performance values $\bar{z}_{i}(i=1,2,3,4)$ of the alternatives $B_{i}(i=1,2,3,4)$, we have $\bar{z}_{1}=\left[\left(s_{2}, 0.3332\right),\left(s_{4},-0.0259\right)\right], \quad \bar{z}_{2}=$ $\left[\left(s_{3},-0.4151\right),\left(s_{4}, 0.2874\right)\right], \bar{z}_{3}=\left[\left(s_{3},-0.3734\right),\left(s_{4}\right.\right.$, $0.1859)], \bar{z}_{4}=\left[\left(s_{3}, 0.1432\right),\left(s_{4}, 0.4915\right)\right]$.

Step 6. Rank all the alternatives and select the best one(s) in accordance with the values of $\bar{z}_{i}(i=$ $1,2,3,4)$.

$$
B_{4} \succ B_{2} \succ B_{3} \succ B_{1}
$$

Thus the best alternative is $B_{4}$.

\section{Conclusions}

We have developed the linguistic interval 2-tuple representation model and some new linguistic aggregation operators, such as linguistic interval 2-tuple power average (LI2TPA) operator, linguistic interval 2-tuple weighted power average (LI2TWPA) operator, linguistic interval 2-tuple power ordered weighted average (LI2TPOWA) operator which are based on PA operator. We have also established some of their properties, such as commutativity, boundedness, etc. Moreover, we have propose two approaches to deal with multiple attribute group decision making problems under linguistic environment. In the situations where the weighting vector of the decision makers is known, we develop an approach which is based on the LI2TWPA operator. On the other hand, in the situations where the weighting vector of the decision makers is unknown, we develop another approach which is based on the LI2TPOWA operator. The prominent advantage of the developed approaches is that they can take all the given arguments and their relationships into account. Therefore, the new approaches can relieve the influence of unduly high or unduly low arguments on the decision results, and thus can make the decision results more reasonable. Finally, a numerical example is provided to illustrate the effectiveness of the new approach.

\section{Acknowledgment}

This work is partially supported by the research fund of Sichuan Key Laboratory of Intelligent Network Information Processing (SGXZD100210), the National Natural Science Foundation (61175055, 61105059), Sichuan Key Technology Research and Development Program (2012GZ0019, 2011FZ0051) and the research fund of education department of Sichuan province (10ZC058) and the Open Research Fund of Key Laboratory of network intelligent information processing,Xihua University(SZJJ2011-021).

\section{References}

1. M. Delgado, F. Herrera, E. Herrera-Viedma, L. Martínez, "Combining numerical and linguistic information in group decision making," Journal of Information Sciences, 107, 177-194 (1998).

2. F.Herrera, E.Herrera-Viedma, J.L.Verdegay, "Linguistic measures based on fuzzy coincidence for reaching consensus in group decision making," International Journal of Approximate Reasoning, 16, 309334 (1997). 


$$
\bar{A}=\left(\begin{array}{cccc}
{\left[\left(s_{2}, 0.4416\right),\left(s_{4},-0.2792\right)\right]} & {\left[\left(s_{2}, 0.4460\right),\left(s_{4}, 0.4460\right)\right]} & {\left[\left(s_{2},-0.1114\right),\left(s_{3}, 0.1741\right)\right]} \\
{\left[\left(s_{2}, 0.2874\right),\left(s_{4}, 0.2874\right)\right]} & {\left[\left(s_{3},-0.2874\right),\left(s_{4}, 0.2874\right)\right]} & {\left[\left(s_{3},-0.2881\right),\left(s_{4}, 0.2874\right)\right]} \\
{\left[\left(s_{1}, 0.4687\right),\left(s_{2}, 0.4687\right)\right]} & {\left[\left(s_{3}, 0.2532\right),\left(s_{5}, 0.1756\right)\right]} & {\left[\left(s_{3},-0.2032\right),\left(s_{4}, 0.2872\right)\right]} \\
{\left[\left(s_{3}, 0.3982\right),\left(s_{5},-0.2979\right)\right]} & {\left[\left(s_{3},-0.1288\right),\left(s_{4}, 0.2626\right)\right]} & {\left[\left(s_{3}, 0.4405\right),\left(s_{5},-0.2522\right)\right]}
\end{array}\right)
$$

3. F. Herrera, E. Herrera-Viedma, "Aggregation operators for linguistic weighted information," IEEE Transactions on Systems, Man, and Cybernetics-Part A: Sys- tems and Humans, 27, 646-656 (1997).

4. F. Herrera, E. Herrera-Viedma, "Linguistic decision analysis: steps for solving decision problems under linguistic information," Fuzzy Sets and Systems, 115, 67-82 (2000).

5. F. Herrera, L. Martínez, "A 2-tuple fuzzy linguistic representation model for computing with words," IEEE Transactions on Fuzzy Systems, 8, 746-752 (2000).

6. F. Herrera, L. Martínez, "A model based on linguistic 2-tuples for dealing with multigranular hierarchical linguistic contexts in multi-expert decisionmaking," IEEE Transactions on Systems, Man, and Cybernetics-Part B: Cybernetics, 31, 227-234 (2001).

7. Z. Pei, D. Ruan, Y. Xu, J. Liu, "Linguistic Values Based Intelligent Information Processing: Theory, Methods, and Applications,", Atlantis press \& World Scientific, 2009.

8. Z. Pei, D. Ruan, Y. Xu, J. Liu, "Handling Linguistic Information over the Internet Based on A Multi-agent System," International Journal of Intelligent Systems, 22(5), 435-453 (2007).

9. Z. Pei, "Fuzzy risk analysis based on linguistic information fusion," ICIC Express Letters, 3(3), 325-330 (2009).

10. Z. Pei, P. Shi, "Fuzzy risk analysis based on linguistic aggregation operators," International Journal of Innovative Computing, Information and Control, 7(12), 7105-7118 (2011).

11. Z. Pei, D. Ruan, J. Liu, Y. Xu, "A linguistic aggregation operator with three kinds of weights for nuclear safeguards evaluation," Knowledge-Based Systems, 28, 19-26 (2012).

12. I. Palomares, J. Liu, Y. Xu, L. Martínez, "Modelling experts' attitudes in group decision making," Soft Computing, 16, 1755-1766 (2012).

13. V. Torra, "The weighted OWA operator," International Journal of Intelligent Systems, 12, 153-166 (1997).

14. C.F. Wei, Z. Pei, H. Li, "An induced OWA operator in coal mine safety evaluation," Journal of Computer and System Sciences, 78(4), 997-1005 (2012).

15. S.P. Wan, "Power average operators of trapezoidal intuitionistic fuzzy numbers and application to multiattribute group decision making," Applied Mathemat- ical Modelling, doi:10.1016/j.apm.2012.09.017.

16. Z.S. Xu, "EOWA and EOWG operators for aggregating linguistic labels based on linguistic preference relations," International Journal of Uncertainty, Fuzziness and Knowledge-Based Systems, 12, 791810 (2004).

17. Z.S. Xu, "Uncertain linguistic aggregation operators based approach to multiple attribute group decision making under uncertain linguistic environment," Information Sciences, 168, 171-184 (2004).

18. Z.S. Xu, "Induced uncertain linguistic OWA operators applied to group decision making," Information $\mathrm{Fu}$ sion, 7, 231-238 (2006).

19. Z.S. Xu, R.R. Yager, "Power-geometric operators and their use in group decision making," IEEE Transactions on Fuzzy Systems, 18, 94-105 (2010).

20. Z.S. Xu, "Approaches to multiple attribute group decision making based on intuitionistic fuzzy power aggregation operators," Knowledge-Based Systems, 24, 749-760 (2011).

21. Z.S. Xu, X.Q. Cai, "Uncertain power average operators for aggregating interval fuzzy preference relations," Group Decision Negotiation, 21, 381-397 (2012).

22. Y.J. Xu, L. Huang, "An approach to group decision making problems based on 2-tuple linguistic aggregation operators," ISECS International Colloquium on Computing, Communication, Control, and Management, 73-77 (2008).

23. Y.J. Xu, H.M. Wang, "Approaches based on 2-tuple linguistic power aggregation operators for multiple attribute group decision making under linguistic environment," Applied Soft Computing, 11, 3988-3997 (2011).

24. Y.J. Xu, J.M. Merigó, H.M. Wang, "Linguistic power aggregation operators and their application to multiple attribute group decision making," Applied Mathematical Modelling, 36, 5427-5444 (2012).

25. R.R. Yager, "On ordered weighted averaging aggregation operators in multi-criteria decision making," IEEE Transactions on Systems, Man, and Cybernetics, 18, 183-190 (1988).

26. R.R. Yager, "Applications and extensions of OWA aggregations," International Journal of Man-Machine Studied, 37, 103-132 (1992).

27. R.R. Yager, “ An approach to ordinal decision making," International Journal of Approximate Reason- 
ing, 12, 237-261 (1995).

28. R.R. Yager, " Quantifier guided aggregation using OWA operators," International Journal of Intelligent Systems, 11, 49-73 (1996).

29. R.R. Yager, A. Rybalov, "Understanding the median as a fusion operator," International Journal of General Systems, 26, 239-263 (1997).

30. R.R. Yager, "Fusion of ordinal information using weighted median aggregation," International Journal of Approximate Reasoning, 18, 35-52 (1998).

31. R.R. Yager, "The power average operator," IEEE Transactions on Systems, Man and Cybernetics A, 31, 724-731 (2001).
32. H.M. Zhang, "The multiattribute group decision making method based on aggregation operators with interval-valued 2-tuple linguistic information," Mathematical and Computer Modelling, 56, 27-35 (2012).

33. L.G. Zhou, H.Y. Chen, J.P. Liu, "Generalized power aggregation operators and their applications in group decision making," Computers \& Industrial Engineering, 62, 989-999 (2012).

34. L.G. Zhou, H.Y. Chen, "A generalization of the power aggregation operators for linguistic environment and its application in group decision making," KnowledgeBased Systems, 26, 216-224 (2012). 